\title{
Stabilized interior penalty methods for the time-harmonic Maxwell equations
}

\author{
I. Perugia $^{\text {a,1 }} \quad$ D. Schötzau ${ }^{\text {b }} \quad$ P. Monk ${ }^{c}$ \\ ${ }^{a}$ Dipartimento di Matematica, Università di Pavia, \\ Via Ferrata 1, 27100 Pavia, Italy, email: perugia@dimat .unipv. it \\ ${ }^{\mathrm{b}}$ School of Mathematics, University of Minnesota, \\ Minneapolis, MN 55455, USA, email: schoetza@math.umn. edu \\ ${ }^{\mathrm{c}}$ Department of Mathematical Sciences, University of Delaware, \\ Newark, DE 19716, USA, email: monk@math.udel.edu
}

\begin{abstract}
We propose stabilized interior penalty discontinuous Galerkin methods for the indefinite time-harmonic Maxwell system. The methods are based on a mixed formulation of the boundary value problem chosen to provide control on the divergence of the electric field. We prove optimal error estimates for the methods in the special case of smooth coefficients and perfectly conducting boundary using a duality approach.
\end{abstract}

Key words: Finite elements, discontinuous Galerkin methods, interior penalty methods, time-harmonic Maxwell's equations

\section{Introduction}

The numerical solution of the time-harmonic Maxwell's equations presents a number of challenges. First, away from boundaries and material interfaces, the solution is smooth and oscillatory. The need to approximate the oscillations requires a sufficiently fine grid compared to the wave-length of the solution, and results in a large number of degrees of freedom if many wavelengths are contained in the domain of interest. This requirement can be loosened (but not entirely avoided) by the use of high order methods [1,2], so that it is desirable to use high order methods where

1 Supported in part by the NSF (Grant DMS-9807491) and by the Supercomputing Institute of the University of Minnesota. This work was carried out when the author was visiting the School of Mathematics, University of Minnesota. 
the solution is smooth. A second problem is that at the boundary of the domain the solution can be singular [3]. Indeed, on a non-convex polyhedral domain the straightforward application of continuous finite element methods can result in a discrete solution that converges to a vector function that is not a solution of Maxwell's equations [4]. While it is possible to modify the variational form to correct for this failure [5], a similar problem also occurs at interfaces between different materials. This is because at discontinuities in the electric properties of the materials in the domain of the electromagnetic field, the electric field is discontinuous. Thus continuous elements need to be modified at such interfaces. A third problem, which we will not discuss here, is the numerical solution of the sparse indefinite matrix problem resulting from the finite element discretization.

Considerations of the first two problems mentioned above have lead to a widespread adoption of edge finite elements [6,7] for the discretization of the timeharmonic Maxwell's equations. For an engineering view of such elements, a good summary is contained in the books [8,9]. An error analysis of these elements has been given in [10-12] and the profound connection between these elements and differential forms has been noted for example in [13-15]. Perhaps the main problem with such elements is that they become rather complex as the order of the elements is increased, and like all conforming methods they require a suitable finite element grid which complicates implementing adaptive solvers. Nevertheless, adaptive $h p$ finite element solvers have been implemented and show considerable promise [16].

In this paper we propose a new way to discretize the indefinite time-harmonic Maxwell system based on a discontinuous Galerkin method (denoted DG in the remainder of the paper). In particular, we propose a suitable extension of the interior penalty methods to the Maxwell system. These methods date back at least to [17-20] and have been studied for coercive elliptic and convection diffusion problems more recently in [21-23]. We mention that several other DG methods for standard coercive elliptic problems can be found in the literature (for instance the LDG method [24,25] or the DG method introduced by Baumann and Oden [26,27]) and unified analyzes of discontinuous methods in the context of elliptic problems have been presented in [28,29]. For the time-harmonic Maxwell equations in the low-frequency regime, where the resulting bilinear forms are coercive, the LDG methods have been recently investigated in [30].

Our goal is to produce a flexible solver in which the order of the scheme can be changed easily between different regions of the grid. In addition we hope to exploit the fact that DG grids do not need to be aligned in order to improve the efficiency of wave propagation of the method. Thus in regions with different electromagnetic properties (and hence different wave speeds), different grid sizes can be used to balance the propagation accuracy of the scheme in each subdomain. Finally it may be possible to "tune" parameters in the DG scheme to improve propagation accuracy (this is certainly possible in 1D one space dimension!). 
In this paper we prove basic error estimates for our proposed schemes under the assumption of smoothly varying material properties. This assumption is needed for certain a-priori estimates used in the analysis. Ultimately we hope to extend these results to more general coefficients and boundary conditions, and we detail the formulation in these cases.

Perhaps the closest approach in the literature to the DG methods we propose is the ultra weak variational method of Cessenat [31]. While successful in practice, this method is still incompletely understood on a theoretical level. For example convergence is not proved for the standard perfectly electrically conducting boundary condition (or near a singularity), or in general throughout the domain of computation. However the successful use of this method is one motivation for proposing the methods in this paper which are convergent globally even in the presence of boundary singularities. Another similar approach is the mortar finite element method applied to the Maxwell equations [32]. To our knowledge, convergence has not been proved for this method in the case of wave propagation. However the success of this method applied to low frequency eddy current problems (in which case the resulting bilinear forms are coercive) suggests that mortar methods or similar domain decomposition methods could be useful for scattering problems [33]. Another domain decomposition approach is the FETI method applied to the Maxwell equations [34]. A Lagrange multiplier based version of this method was analyzed in [35] for the coercive Maxwell problem arising in time stepping. Again to our knowledge, convergence has not been proved for this method in the case of wave propagation in Maxwell's equations.

The outline of our paper is as follows. In section 2, we start by detailing the mixed formulation we shall use as the basis of the DG methods proposed here. We also summarize some regularity and existence results. Then in section 3 we propose the DG methods that are the subject of this paper. The main result of the paper is an optimal a priori error bound that we present in section 4. Its proof is based on a duality approach and is contained in section 5 and section 6 . We end our presentation with some concluding remarks in section 7 .

\section{A mixed formulation for the time-harmonic Maxwell equations}

In this section, we introduce the time-harmonic Maxwell equations and present a mixed formulation for the continuous problem which will be the basis for the DG methods introduced here. 


\subsection{Time-harmonic Maxwell's equations}

Let $\Omega$ be a bounded Lipschitz polyhedron in $\mathbb{R}^{3}$ with connected boundary $\partial \Omega$. The model problem we shall consider is to compute a time-harmonic electric field $\mathcal{E}$ in the cavity $\Omega$ with perfectly conducting boundary. Let $\omega$ denote the temporal frequency of the time-harmonic field so that the corresponding time dependent field $\mathbf{E}$ at position $\mathbf{x} \in \Omega$ and time $t$ is given by

$$
\mathbf{E}(\mathbf{x}, t)=\Re(\mathcal{E}(\mathbf{x}) \exp (-i \omega t)) .
$$

Then $\mathcal{E}: \Omega \rightarrow \mathbb{C}^{3}$ satisfies the Maxwell system

$$
\nabla \times \mu_{r}^{-1} \nabla \times \mathcal{E}-k^{2} \varepsilon_{r} \mathcal{E}=\mathcal{J} \quad \text { in } \Omega
$$

where $\mu_{r}$ is the relative magnetic permeability and $\varepsilon_{r}$ is the relative electric permittivity of the medium in the cavity $\Omega$. We assume that $\mu_{r}$ and $\varepsilon_{r}$ are real, smooth, and uniformly positive functions of the position in $\Omega$. In addition the real wave number $k$ is given by

$$
k=\omega \sqrt{\varepsilon_{0} \mu_{0}},
$$

where $\mu_{0}$ is the magnetic permeability and $\varepsilon_{0}$ is the electric permittivity of free space. The source function $\mathcal{J}$ is related to the applied current density driving the cavity and is assumed to be a given vector function in $L^{2}(\Omega)^{3}$.

The assumption that $\Omega$ has a perfectly conducting boundary gives the following boundary condition on $\partial \Omega$ :

$$
\mathbf{n} \times \mathcal{E}=\mathbf{0} \quad \text { on } \partial \Omega
$$

Here $\mathbf{n}$ denotes the outward normal unit vector to $\partial \Omega$.

Throughout the paper, we will assume that $k^{2}$ is not an interior Maxwell eigenvalue (see also Proposition 1 below), i.e., for any $\mathcal{E} \neq \mathbf{0}$, the pair $\left(\lambda=k^{2}, \mathcal{E}\right)$ is not an eigensolution of the problem $\nabla \times \mu_{r}^{-1} \nabla \times \mathcal{E}=\lambda \varepsilon_{r} \mathcal{E}$ in $\Omega, \mathbf{n} \times \mathcal{E}=\mathbf{0}$ on $\partial \Omega$. Note that this assumption would not be necessary if some region of $\Omega$ (containing a ball of non-zero radius) had a non zero conductivity which would imply that the imaginary part of $\varepsilon_{r}$ is positive there. Note also that in the special case considered here the real and imaginary parts of the solution decouple, and hence we can assume that $\mathcal{E}$ is real. If $\varepsilon_{r}$ is complex valued or if impedance boundary conditions are imposed, the real and imaginary parts are coupled.

\subsection{Mixed formulation}

Our DG method is based on a mixed formulation of the Maxwell boundary value problem (1)-(2). Such formulations have been used previously for edge element 
discretizations of Maxwell's equations to improve stability [16], and to handle coercive problems in which meshes are not aligned at a material boundary [35]. We can derive this formulation by using a Helmholtz decomposition.

Given a domain $D$ in $\mathbb{R}^{2}$ or $\mathbb{R}^{3}$, we denote by $H^{s}(D)^{d}, d=1,2,3$, the Sobolev space of real or complex scalar- or vector-valued functions with regularity exponent $s \geq 0$, endowed with the usual norm $\|\cdot\|_{s, D}$ and seminorm $|\cdot|_{s, D}$. We write $H_{0}^{1}(D)$ for the subspace of $H^{1}(D)$ of functions with zero trace and set $L^{2}(D)^{d}=H^{0}(D)^{d}$. For the computational domain $\Omega \subset \mathbb{R}^{3}$, we let

$$
\begin{aligned}
H\left(\operatorname{div}_{\varepsilon_{r}}^{0} ; \Omega\right) & =\left\{\mathbf{u} \in L^{2}(\Omega)^{3} \mid \nabla \cdot\left(\varepsilon_{r} \mathbf{u}\right)=0 \text { in } \Omega\right\} \\
H\left(\operatorname{div}^{0} ; \Omega\right) & =\left\{\mathbf{u} \in L^{2}(\Omega)^{3} \mid \nabla \cdot \mathbf{u}=0 \text { in } \Omega\right\},
\end{aligned}
$$

equipped with the $L^{2}(\Omega)^{3}$-norm. We shall also need to use the standard spaces

$$
\begin{aligned}
H(\operatorname{curl} ; \Omega) & =\left\{\mathbf{u} \in L^{2}(\Omega)^{3} \mid \nabla \times \mathbf{u} \in L^{2}(\Omega)^{3}\right\} \\
H_{0}(\operatorname{curl} ; \Omega) & =\{\mathbf{u} \in H(\operatorname{curl} ; \Omega) \mid \mathbf{n} \times \mathbf{u}=\mathbf{0} \text { on } \partial \Omega\},
\end{aligned}
$$

endowed with the norm

$$
\|\mathbf{u}\|_{\mathrm{curl}, \Omega}^{2}=\|\mathbf{u}\|_{0, \Omega}^{2}+\|\nabla \times \mathbf{u}\|_{0, \Omega}^{2} .
$$

Furthermore, let $L_{\varepsilon_{r}}^{2}(\Omega)^{3}$ denote the space of square integrable functions on $\Omega$ equipped with the inner product

$$
(\mathbf{u}, \mathbf{v})_{\varepsilon_{r}}=\int_{\Omega} \varepsilon_{r} \mathbf{u} \cdot \mathbf{v} d \mathbf{x} .
$$

We consider the $L_{\varepsilon_{r}}^{2}(\Omega)^{3}$-orthogonal Helmholtz decomposition of the function $\mathcal{E} \in$ $H_{0}(\operatorname{curl} ; \Omega)$ given by $\mathcal{E}=\mathbf{u}+\nabla p$, where $\mathbf{u} \in H_{0}(\operatorname{curl} ; \Omega) \cap H\left(\operatorname{div}_{\varepsilon_{r}}^{0} ; \Omega\right)$ and $p \in H_{0}^{1}(\Omega)$, see [36].

Using this decomposition, problem (1)-(2) can be reformulated as follows: find $\mathbf{u} \in H(\operatorname{curl} ; \Omega)$ and $p \in H^{1}(\Omega)$ such that

$$
\begin{aligned}
\nabla \times \mu_{r}^{-1} \nabla \times \mathbf{u}-k^{2} \varepsilon_{r} \mathbf{u}-k^{2} \varepsilon_{r} \nabla p & =\mathcal{J} & & \text { in } \Omega \\
\nabla \cdot\left(\varepsilon_{r} \mathbf{u}\right) & =0 & & \text { in } \Omega \\
\mathbf{n} \times \mathbf{u} & =\mathbf{0} & & \text { on } \partial \Omega \\
p & =0 & & \text { on } \partial \Omega .
\end{aligned}
$$

We start by showing well-posedness of problem (3)-(6).

Proposition 1 Assume that $k^{2}$ is not a Maxwell eigenvalue. Then problem (3)-(6) has a unique solution $(\mathbf{u}, p) \in H_{0}(\operatorname{curl} ; \Omega) \cap H\left(\operatorname{div}_{\varepsilon_{r}}^{0} ; \Omega\right) \times H_{0}^{1}(\Omega)$, with $\mu_{r}^{-1} \nabla \times$ $\mathbf{u} \in H(\mathrm{curl} ; \Omega)$, and we have the stability estimates

$$
\|\mathbf{u}\|_{\text {curl }, \Omega} \leq C_{\text {stab }}\|\mathcal{J}\|_{0, \Omega}, \quad\|p\|_{1, \Omega} \leq k^{-2} C_{\text {ell }}\|\mathcal{J}\|_{0, \Omega},
$$


with positive constants $C_{\mathrm{stab}}$ and $C_{\mathrm{ell}}, C_{\mathrm{ell}}$ independent of $k$.

Proof Consider the Helmholtz decomposition of $\mathcal{J}$ as a function in $L^{2}(\Omega)^{3}, \mathcal{J}=$ $\mathbf{J}_{0}+\nabla j$ with $\mathbf{J}_{0} \in H\left(\operatorname{div}^{0} ; \Omega\right)$ and $j \in H_{0}^{1}(\Omega)$. Owing to the orthogonality of this decomposition, problem (3)-(6) decouples into two independent subproblems, namely into the Maxwell problem with divergence free data

$$
\begin{aligned}
\nabla \times \mu_{r}^{-1} \nabla \times \mathbf{u}-k^{2} \varepsilon_{r} \mathbf{u} & =\mathbf{J}_{0} & & \text { in } \Omega \\
\nabla \cdot\left(\varepsilon_{r} \mathbf{u}\right) & =0 & & \text { in } \Omega \\
\mathbf{n} \times \mathbf{u} & =\mathbf{0} & & \text { on } \partial \Omega
\end{aligned}
$$

and the elliptic problem

$$
-k^{2} \nabla \cdot\left(\varepsilon_{r} \nabla p\right)=F \quad \text { in } \Omega, \quad p=0 \quad \text { on } \partial \Omega,
$$

with right hand side $F \in H^{-1}(\Omega)$ defined by $F(q)=-\int_{\Omega} \nabla j \cdot \nabla q d \mathbf{x}$, for all $q \in H_{0}^{1}(\Omega)$. Existence and uniqueness of solutions to (7) follow now in a standard way from Fredholm theory and the coercivity of the form $\int_{\Omega} \mu_{r}^{-1} \nabla \times \mathbf{u} \cdot \nabla \times \mathbf{v} d \mathbf{x}$ on the space $H_{0}(\operatorname{curl} ; \Omega) \cap H\left(\operatorname{div}_{\varepsilon_{r}}^{0} ; \Omega\right)$ (see [36, Proposition 2.7]). Furthermore, $\mu_{r}^{-1} \nabla \times \mathbf{u} \in H(\operatorname{curl} ; \Omega)$ and

$$
\|\mathbf{u}\|_{\text {curl }, \Omega} \leq C_{\text {stab }}\left\|\mathbf{J}_{0}\right\|_{0, \Omega} \leq C_{\text {stab }}\|\mathcal{J}\|_{0, \Omega}
$$

with a stability constant $C_{\text {stab }}>0$ depending on $\Omega, \mu_{r}, \varepsilon_{r}$ and on the wave number $k^{2}$. For problem (8), existence and uniqueness follows from standard elliptic theory, and we have

$$
\|p\|_{1, \Omega} \leq k^{-2} C_{\text {ell }}\|F\|_{-1, \Omega} \leq k^{-2} C_{\text {ell }}\|\nabla j\|_{0, \Omega} \leq k^{-2} C_{\text {ell }}\|\mathcal{J}\|_{0, \Omega},
$$

with $C_{\text {ell }}>0$ only depending on $\Omega$ and $\varepsilon_{r}$.

In our duality approach in section 6 we will also make use of the following regularity result.

Proposition 2 For smooth coefficients $\mu_{r}$ and $\varepsilon_{r}$, there exists a regularity exponent $\sigma=\sigma(\Omega)>\frac{1}{2}$ such that the solution $\mathbf{u}$ in (3)-(6) satisfies $\mathbf{u} \in H^{\sigma}(\Omega)^{3}$ and $\nabla \times \mathbf{u} \in H^{\sigma}(\Omega)^{3}$. Furthermore,

$$
\|\mathbf{u}\|_{\sigma, \Omega}+\|\nabla \times \mathbf{u}\|_{\sigma, \Omega} \leq C_{\mathrm{reg}}\|\mathcal{J}\|_{0, \Omega}
$$

with a positive constant $C_{\mathrm{reg}}$ depending on $k^{2}, \mu_{r}$ and $\varepsilon_{r}$.

Proof By decoupling problem (3)-(6) into (7) and (8), we see that the solution $\mathbf{u}$ of (7) satisfies $\nabla \times \mathbf{u} \in L^{2}(\Omega)^{3}, \nabla \cdot \mathbf{u} \in L^{2}(\Omega)^{3}$ (here we use the assumption that $\varepsilon_{r}$ is smooth, so that $\varepsilon_{r} \nabla \cdot \mathbf{u}=\nabla \cdot\left(\varepsilon_{r} \mathbf{u}\right)-\nabla \varepsilon_{r} \cdot \mathbf{u}$ holds true) and $\mathbf{n} \times \mathbf{u}=\mathbf{0}$ on $\partial \Omega$. From [37, Proposition 3.7], it follows that $\mathbf{u} \in H^{\sigma_{1}}(\Omega)^{3}$ for $\sigma_{1}>\frac{1}{2}$ and 
$\|\mathbf{u}\|_{\sigma_{1}, \Omega} \leq C(\Omega)\|\mathbf{u}\|_{\text {curl }, \Omega}$ with an embedding constant $C(\Omega)$ just depending on $\Omega$. Thus, from Proposition 1, we have $\|\mathbf{u}\|_{\sigma_{1}, \Omega} \leq C_{1}\|\mathcal{J}\|_{0, \Omega}$.

Now set $\mathbf{w}=\mu_{r}^{-1} \nabla \times \mathbf{u}$. From the first equation in (7), we have $\nabla \times \mathbf{w}=\mathbf{J}_{0}+$ $k^{2} \varepsilon_{r} \mathbf{u} \in L^{2}(\Omega)^{3}$. Furthermore, $\nabla \cdot\left(\mu_{r} \mathbf{w}\right)=0$ and $\mu_{r} \mathbf{w} \cdot \mathbf{n}=\nabla \times \mathbf{u} \cdot \mathbf{n}=0$ on $\partial \Omega$. Since $\mu_{r}$ is smooth, using again [37, Proposition 3.7], it follows that $\mathbf{w} \in H^{\sigma_{2}}(\Omega)^{3}$ for $\sigma_{2}>\frac{1}{2}$ and $\|\mathbf{w}\|_{\sigma_{2}, \Omega} \leq C(\Omega)\|\mathbf{w}\|_{\text {curl }, \Omega} \leq C\left(\Omega, \mu_{r}\right)\left(\|\nabla \times \mathbf{u}\|_{0, \Omega}^{2}+\| \mathbf{J}_{0}+\right.$ $\left.k^{2} \varepsilon_{r} \mathbf{u} \|_{0, \Omega}^{2}\right)^{\frac{1}{2}}$. Hence, from the triangle inequality and Proposition 1, we conclude that $\|\mathbf{w}\|_{\sigma_{2}, \Omega} \leq C_{2}\|\mathcal{J}\|_{0, \Omega}$. Choosing $\sigma:=\min \left\{\sigma_{1}, \sigma_{2}\right\}$ and $C_{\text {reg }}=\max \left\{C_{1}, C_{2}\right\}$ completes the proof.

Remark 3 If the polyhedron $\Omega$ is convex and $\mu_{r}=\varepsilon_{r}=1$, the parameter $\sigma$ in Proposition 2 can be chosen as $\sigma=1$, see [37].

\section{Discontinuous Galerkin discretization}

In this section, we introduce stabilized interior penalty discontinuous Galerkin discretizations for the Maxwell system (3)-(6).

\subsection{Triangulations}

Let $\mathcal{T}_{h}$ be a regular triangulation of the domain $\Omega$ into tetrahedra. We denote by $h_{K}$ the diameter of the element $K$ and set $h=\max _{K \in \mathcal{T}_{h}} h_{K}$. The diameter of the face $f$ is denoted by $h_{f}$. We also assume the triangulation to be shape regular, that is, there is a positive constant $\kappa$ such that, for any $K \in \mathcal{T}_{h}$,

$$
\frac{h_{K}}{\rho_{K}} \leq \kappa,
$$

where $\rho_{K}$ is the diameter of the biggest ball contained in $K$ (see [38, p. 124]).

Let $\mathcal{E}$ be the union of all the faces of $\mathcal{T}_{h}$, and $\mathcal{E}_{\mathcal{I}}$ the union of the internal faces. We define the function $\mathrm{h}$ in $L^{\infty}(\mathcal{E})$ by

$$
\mathrm{h}=\mathrm{h}(\mathbf{x})=h_{f} \quad \text { if } \mathbf{x} \in f .
$$

\subsection{Trace operators}

First, we need to define some notation concerning functions in $H^{s}\left(\mathcal{T}_{h}\right):=\{v$ : $\left.\left.v\right|_{K} \in H^{s}(K), K \in \mathcal{T}_{h}\right\}$, for $s>\frac{1}{2}$. The elementwise traces of such functions 
belong to $\operatorname{TR}(\mathcal{E}):=\Pi_{K \in \mathcal{T}_{h}} L^{2}(\partial K)$; they are double-valued on $\mathcal{E}_{\mathcal{I}}$ and singlevalued on $\mathcal{E} \backslash \mathcal{E}_{\mathcal{I}}$. The space $L^{2}(\mathcal{E})$ can be identified with the functions in $\operatorname{TR}(\mathcal{E})$ for which the two trace values coincide.

Next, we introduce certain trace operators. To this end, fix $\mathbf{w} \in \operatorname{TR}(\mathcal{E})^{3}$ and $\varphi \in$ $\operatorname{TR}(\mathcal{E})$, and let $e \subset \mathcal{E}_{\mathcal{I}}$ be an interior face shared by the elements $K_{1}$ and $K_{2}$. Let $\mathbf{n}_{i}$ be the normal unit vector pointing exterior to $K_{i}$ and $\mathbf{w}_{i}=\left.\mathbf{w}\right|_{\partial K_{i}}, \varphi_{i}=\left.\varphi\right|_{\partial K_{i}}$ $(i=1,2)$. Then we define for $\mathbf{x} \in e$ the average, the tangential jump and the normal jump of $\mathbf{w}$ as follows:

$$
\{\mathbf{w}\}=\frac{1}{2}\left(\mathbf{w}_{1}+\mathbf{w}_{2}\right) \quad \llbracket \mathbf{w} \rrbracket_{T}=\mathbf{n}_{1} \times \mathbf{w}_{1}+\mathbf{n}_{2} \times \mathbf{w}_{2} \quad \llbracket \mathbf{w} \rrbracket_{N}=\mathbf{w}_{1} \cdot \mathbf{n}_{1}+\mathbf{w}_{2} \cdot \mathbf{n}_{2} .
$$

Similarly, we define for $\mathbf{x} \in e$ the average and the normal jump of $\varphi$ by

$$
\left\{\{\varphi\}=\frac{1}{2}\left(\varphi_{1}+\varphi_{2}\right) \quad \llbracket \varphi \rrbracket_{N}=\varphi_{1} \mathbf{n}_{1}+\varphi_{2} \mathbf{n}_{2}\right.
$$

Then, on any boundary face $e \subset \mathcal{E} \backslash \mathcal{E}_{\mathcal{I}}$, we set for $\mathbf{x} \in e$

$$
\left\{\{\mathbf{w}\}=\mathbf{w} \quad \llbracket \mathbf{w} \rrbracket_{T}=\mathbf{n} \times \mathbf{w} \quad \llbracket \varphi \rrbracket_{N}=\varphi \mathbf{n} .\right.
$$

Since we will not require either of the quantities $\{\varphi \varphi\}$ and $\llbracket \mathbf{w} \rrbracket_{N}$ on the boundary $\mathcal{E} \backslash \mathcal{E}_{\mathcal{I}}$, we leave them undefined.

If $\mathbf{w} \in H(\operatorname{curl} ; \Omega)$, then, for all $e \subset \mathcal{E}_{\mathcal{I}}$, the jump condition $\mathbf{n}_{1} \times \mathbf{w}_{1}+\mathbf{n}_{2} \times \mathbf{w}_{2}=\mathbf{0}$ holds true in $H_{00}^{-\frac{1}{2}}(e)^{3}$, and thus also in $L^{2}(e)^{3}$ (for the definition of $H_{00}^{-\frac{1}{2}}(e)$, see, e.g., [39]). Therefore, $\llbracket \mathbf{w} \rrbracket_{T}$ is equal to zero on $\mathcal{E}_{\mathcal{I}}$. Similarly, for $\mathbf{w} \in H(\operatorname{div} ; \Omega)$, we have that $\llbracket \mathbf{w} \rrbracket_{N}$ is well-defined and equal to zero on $\mathcal{E}_{\mathcal{I}}$. Furthermore, for the exact solution $\mathbf{u} \in H_{0}(\operatorname{curl} ; \Omega) \cap H\left(\operatorname{div}_{\varepsilon_{r}}^{0} ; \Omega\right)$, we have $\llbracket \mathbf{u} \rrbracket_{T}=\mathbf{0}$ in $L^{2}(e)^{3}$ for any boundary face $e$, in addition to $\llbracket \mathbf{u} \rrbracket_{T}=\mathbf{0}$ and $\llbracket \varepsilon_{r} \mathbf{u} \rrbracket_{N}=0$ on $\mathcal{E}_{\mathcal{I}}$.

\subsection{Stabilized interior penalty discontinuous Galerkin methods}

We approximate $\mathbf{u}$ and $p$ in the discontinuous finite element space $\mathbf{V}_{h} \times Q_{h}$ where

$$
\begin{aligned}
& \mathbf{V}_{h}=\left\{\mathbf{v} \in L^{2}(\Omega)^{3}:\left.\mathbf{v}\right|_{K} \in \mathcal{P}^{\ell}(K)^{3}, \forall K \in \mathcal{T}_{h}\right\} \\
& Q_{h}=\left\{q \in L^{2}(\Omega):\left.q\right|_{K} \in \mathcal{P}^{\ell}(K), \forall K \in \mathcal{T}_{h}\right\}
\end{aligned}
$$

for an approximation order $\ell \geq 1$, with $\mathcal{P}^{\ell}(K)$ denoting the space of polynomials of degree at most $\ell$ on $K$.

We consider the following discontinuous Galerkin method: find $\left(\mathbf{u}_{h}, p_{h}\right) \in \mathbf{V}_{h} \times Q_{h}$ 
such that, for any $(\mathbf{v}, q) \in \mathbf{V}_{h} \times Q_{h}$,

$$
\begin{aligned}
& a\left(\mathbf{u}_{h}, \mathbf{v}\right)+c\left(\mathbf{u}_{h}, \mathbf{v}\right)+d\left(\mathbf{u}_{h}, \mathbf{v}\right)-k^{2}\left(\mathbf{u}_{h}, \mathbf{v}\right)_{\varepsilon_{r}}+b\left(\mathbf{v}, p_{h}\right)=\mathcal{F}(\mathbf{v}) \\
& b\left(\mathbf{u}_{h}, q\right)-e\left(p_{h}, q\right) \\
& =0 .
\end{aligned}
$$

Here,

$$
\begin{aligned}
& a(\mathbf{u}, \mathbf{v})=\int_{\Omega} \mu_{r}^{-1} \nabla_{h} \times \mathbf{u} \cdot \nabla_{h} \times \mathbf{v} d \mathbf{x}-\int_{\mathcal{E}} \llbracket \mathbf{u} \rrbracket_{T} \cdot\left\{\left\{\mu_{r}^{-1} \nabla_{h} \times \mathbf{v}\right\} d s\right. \\
& -\int_{\mathcal{E}} \llbracket \mathbf{v} \rrbracket_{T} \cdot\left\{\left\{\mu_{r}^{-1} \nabla_{h} \times \mathbf{u}\right\}\right\} d s \\
& \left.b(\mathbf{v}, p)=k^{2} \int_{\Omega} p \nabla_{h} \cdot\left(\varepsilon_{r} \mathbf{v}\right) d \mathbf{x}-k^{2} \int_{\mathcal{E}_{\mathcal{I}}}\{p\}\right\} \llbracket \varepsilon_{r} \mathbf{v} \rrbracket_{N} d s \\
& c(\mathbf{u}, \mathbf{v})=\alpha \int_{\mathcal{E}} \mathrm{h}^{-1} \mathrm{~m}^{-1} \llbracket \mathbf{u} \rrbracket_{T} \cdot \llbracket \mathbf{v} \rrbracket_{T} d s \\
& d(\mathbf{u}, \mathbf{v})=k^{2} \beta \int_{\mathcal{E}_{\mathcal{I}}} \mathrm{h} \llbracket \varepsilon_{r} \mathbf{u} \rrbracket_{N} \llbracket \varepsilon_{r} \mathbf{v} \rrbracket_{N} d s \\
& +k^{2} \beta \sum_{K \in \mathcal{T}_{h}} h_{K}^{2} \int_{K} \nabla_{h} \cdot\left(\varepsilon_{r} \mathbf{u}\right) \nabla_{h} \cdot\left(\varepsilon_{r} \mathbf{v}\right) d \mathbf{x} \\
& e(p, q)=k^{2} \gamma \int_{\mathcal{E}} \mathrm{h}^{-1} \mathrm{e} \llbracket p \rrbracket_{N} \cdot \llbracket q \rrbracket_{N} d s+\alpha \int_{\mathcal{E}} \mathrm{h}^{-1} \mathrm{~m}^{-1} \llbracket \nabla_{h} p \rrbracket_{T} \cdot \llbracket \nabla_{h} q \rrbracket_{T} d s,
\end{aligned}
$$

with $\nabla_{h} \times, \nabla_{h}$ and $\nabla_{h}$ denoting the elementwise curl, divergence and gradient, respectively, and the functions $m$ and e are defined on $\mathcal{E}$ as the restriction to $\mathcal{E}$ of $\mu_{r}$ and $\varepsilon_{r}$, respectively. The parameters $\alpha, \beta$ and $\gamma$ in the forms $c, d$, and $e$ are positive. The purpose of these forms is to stabilize the method. The functional $\mathcal{F}$ at right-hand side of (9) is

$$
\mathcal{F}(\mathbf{v})=\int_{\Omega} \mathcal{J} \cdot \mathbf{v} d \mathbf{x}
$$

Let us discuss the following points about this method:

- The form $a(\cdot, \cdot)+c(\cdot, \cdot)$ corresponds to the interior penalty discretization of the curl-curl operator, cf. [28]; it is symmetric and stable provided that the parameter $\alpha$ is large enough (see Lemma 14 below). The nonsymmetric variant of the interior penalty discretization is obtained by replacing $a$ by

$$
\begin{aligned}
a(\mathbf{u}, \mathbf{v})= & \int_{\Omega} \mu_{r}^{-1} \nabla_{h} \times \mathbf{u} \cdot \nabla_{h} \times \mathbf{v} d \mathbf{x}+\int_{\mathcal{E}} \llbracket \mathbf{u} \rrbracket_{T} \cdot\left\{\mu_{r}^{-1} \nabla_{h} \times \mathbf{v}\right\} d s \\
& -\int_{\mathcal{E}} \llbracket \mathbf{v} \rrbracket_{T} \cdot\left\{\left\{\mu_{r}^{-1} \nabla_{h} \times \mathbf{u}\right\}\right] d s
\end{aligned}
$$

Then the form $a(\cdot, \cdot)+c(\cdot, \cdot)$ is nonsymmetric, but stable for any $\alpha>0$ (see $\operatorname{Re}$ mark 15 below). In the following we will only present the analysis for the symmetric method in (9)-(10), but emphasize that the error estimates so obtained hold true verbatim for its nonsymmetric variant.

- The form $b(\cdot, \cdot)$ discretizes the divergence constraint in the mixed formulation (3)-(6) by means of DG techniques, similar to the forms used in [40] for the Stokes 
system. Notice that, after integration by parts, the form $b(\cdot, \cdot)$ can also be expressed by

$$
b(\mathbf{v}, p)=-k^{2} \int_{\Omega} \varepsilon_{r} \mathbf{v} \cdot \nabla_{h} p d \mathbf{x}+k^{2} \int_{\mathcal{E}} \llbracket p \rrbracket_{N} \cdot\left\{\left\{\varepsilon_{r} \mathbf{v}\right\} d s, \quad(\mathbf{v}, p) \in \mathbf{V}_{h} \times Q_{h} .\right.
$$

- The forms $d(\cdot, \cdot)$ and $e(\cdot, \cdot)$ provide stabilization. While $d(\cdot, \cdot)$ is related to the divergence constraint, the form $e(\cdot, \cdot)$ provides stability via control of jumps of the scalar potential $p$. We found it necessary to include these forms in order to be able to prove optimal error estimates with our techniques of analysis. Whether or not similar results can actually be obtained without these stabilization forms remains an open question and will be investigated numerically in a forthcoming work.

- By elementary manipulations the third term in the form $a(\cdot, \cdot)$ can be expressed by

$$
\begin{aligned}
-\int_{\mathcal{E}} \llbracket \mathbf{v} \rrbracket_{T} \cdot\left\{\left\{\mu_{r}^{-1} \nabla_{h} \times \mathbf{u}\right\}\right\} d s= & \sum_{K \in K} \int_{\partial K} \mathbf{v} \cdot \mathbf{n}_{K} \times\left[\mu_{r}^{-1} \nabla_{h} \times \mathbf{u}\right] d s \\
& -\int_{\mathcal{E}_{\mathcal{I}}}\{\{\mathbf{v}\}] \cdot \llbracket \mu_{r}^{-1} \nabla_{h} \times \mathbf{u} \rrbracket_{T} d s
\end{aligned}
$$

for all $\mathbf{u}, \mathbf{v} \in \mathbf{V}_{h}$, where $\mathbf{n}_{K}$ is the outward normal unit vector to $\partial K$. For the exact solution $\mathbf{u}$ we have $\mathbf{u} \in H_{0}(\operatorname{curl} ; \Omega)$ and $\mu_{r}^{-1} \nabla \times \mathbf{u} \in H(\operatorname{curl} ; \Omega)$. Thus, $\llbracket \mathbf{u} \rrbracket_{T}=\mathbf{0}$ on $\mathcal{E}$ and $\llbracket \mu_{r}^{-1} \nabla_{h} \times \mathbf{u} \rrbracket_{T}=\mathbf{0}$ on $\mathcal{E}_{\mathcal{I}}$, and, with (12), $a(\mathbf{u}, \mathbf{v})$ has to be understood as

$$
a(\mathbf{u}, \mathbf{v})=\int_{\Omega} \mu_{r}^{-1} \nabla_{h} \times \mathbf{u} \cdot \nabla_{h} \times \mathbf{v} d \mathbf{x}+\sum_{K \in K} \int_{\partial K} \mathbf{v} \cdot \mathbf{n}_{K} \times\left[\mu_{r}^{-1} \nabla_{h} \times \mathbf{u}\right] d s
$$

for $\mathbf{v} \in \mathbf{V}_{h}$, where the boundary integrals are in fact duality pairings.

Let us now address the consistency of the method.

Proposition 4 The discontinuous Galerkin method in (9)-(10) is consistent, i.e., the exact solution $(\mathbf{u}, p)$ of problem (3)-(6) satisfies (9)-(10), for all test functions $(\mathbf{v}, q) \in \mathbf{V}_{h} \times Q_{h}$.

Proof We have $\mathbf{u} \in H_{0}(\operatorname{curl} ; \Omega), \mu_{r}^{-1} \nabla \times \mathbf{u} \in H(\operatorname{curl} ; \Omega)$ and $\mathbf{u} \in H\left(\operatorname{div}_{\varepsilon_{r}}^{0} ; \Omega\right)$. Thus, $\llbracket \mathbf{u} \rrbracket_{T}=\mathbf{0}$ on $\mathcal{E}$, as well as $\llbracket \varepsilon_{r} \mathbf{u} \rrbracket_{N}=0$ and $\llbracket \mu_{r}^{-1} \nabla_{h} \times \mathbf{u} \rrbracket_{T}=\mathbf{0}$ on $\mathcal{E}_{\mathcal{I}}$. Moreover, $p \in H_{0}^{1}(\Omega)$ and $\nabla p \in H_{0}(\operatorname{curl} ; \Omega)$ and thus $\llbracket p \rrbracket_{N}=0$ and $\llbracket \nabla p \rrbracket_{T}=\mathbf{0}$ on $\mathcal{E}$. The second equation (10) is then trivially satisfied for all $q \in Q_{h}$. From (12), the first equation (9) reduces to

$$
\begin{aligned}
& \int_{\Omega} \mu_{r}^{-1} \nabla \times \mathbf{u} \cdot \nabla_{h} \times \mathbf{v} d \mathbf{x}+\sum_{K \in K} \int_{\partial K} \mathbf{v} \cdot \mathbf{n}_{K} \times\left[\mu_{r}^{-1} \nabla_{h} \times \mathbf{u}\right] d s \\
& -k^{2} \int_{\Omega} \varepsilon_{r} \mathbf{u} \cdot \mathbf{v} d \mathbf{x}+k^{2} \int_{\Omega} p \nabla_{h} \cdot\left(\varepsilon_{r} \mathbf{v}\right) d \mathbf{x}-k^{2} \int_{\mathcal{E}_{\mathcal{I}}}\left\{[p\} \llbracket \llbracket \varepsilon_{r} \mathbf{v} \rrbracket_{N} d s=\int_{\Omega} \mathcal{J} \cdot \mathbf{v} d \mathbf{x}\right.
\end{aligned}
$$


for $\mathbf{v} \in \mathbf{V}_{h}$. Integration by parts over each element $K$, taking into account the boundary condition for $\mathbf{u}$ and $p$, yields

$$
\int_{\Omega}\left(\nabla \times \mu_{r}^{-1} \nabla \times \mathbf{u}-k^{2} \varepsilon_{r} \mathbf{u}-k^{2} \varepsilon_{r} \nabla p\right) \cdot \mathbf{v} d \mathbf{x}=\int_{\Omega} \mathcal{J} \cdot \mathbf{v} d \mathbf{x}
$$

which is satisfied for all $\mathbf{v} \in \mathbf{V}_{h}$.

Remark 5 Note that the regularity of the solution stated in Proposition 2 as consequence of the smoothness assumption on the coefficients is needed neither in the definition of the method, nor in the proof of Proposition 4. As a matter of fact, the method is defined and consistent for piecewise smooth coefficients $\mu_{r}$ and $\varepsilon_{r}$. In this case, the functions $\mathrm{m}$ and e have to be adjusted by taking the corresponding averages, i.e., by taking $\mathrm{m}=\left\{\left\{\mu_{r}\right\}\right.$ and $\mathrm{e}=\left\{\left\{\varepsilon_{r}\right\}\right.$ on $\mathcal{E}$.

Remark 6 The analysis developed in the following sections makes use of conforming projection operators, and therefore only covers the case of meshes that do not contain hanging nodes. On the other hand, the DG method is well-defined for general non-matching grids. In this case, the interior faces are understood as the (non-empty) interiors of the intersections between two adjacent elements and the function $\mathrm{h}$ has to be redefined on $\mathcal{E}$ as

$$
\mathrm{h}=\mathrm{h}(\mathbf{x})=\left\{\begin{array}{cl}
\frac{1}{2}\left(h_{K}+h_{K^{\prime}}\right) & \text { if } \mathbf{x} \in e=\partial K \cap \partial K^{\prime} \\
h_{K} & \text { if } \mathbf{x} \in e \subset \partial K \cap \partial \Omega .
\end{array}\right.
$$

\section{The main result}

In this section, we present and discuss our main result - an optimal a priori error estimate for the DG method in (9)-(10). The proof of this bound is developed in section 5 and section 6; it is based on a suitable duality argument that heavily relies on the regularity result of Proposition 2, and therefore the assumption of smooth coefficients $\mu_{r}$ and $\varepsilon_{r}$ is essential. Moreover, in order to simplify the presentation, we restrict ourselves to the case $\mu_{r}=\varepsilon_{r}=1$. The extension to general smooth coefficients is straightforward.

Define the spaces

$$
\mathbf{V}(h):=\mathbf{V}_{h}+\left(H_{0}(\operatorname{curl} ; \Omega) \cap H\left(\operatorname{div}^{0} ; \Omega\right)\right) \quad Q(h):=Q_{h}+H_{0}^{1}(\Omega)
$$

and the broken norm $\|(\mathbf{u}, p)\|_{h}$ given by

$$
\|(\mathbf{u}, p)\|_{h}^{2}=k^{2}\|\mathbf{u}\|_{0, \Omega}^{2}+k^{2}\left\|\nabla_{h} p\right\|_{0, \Omega}^{2}+|(\mathbf{u}, p)|_{h}^{2},
$$


where the seminorm $|(\mathbf{u}, p)|_{h}$ is given by

$$
\begin{aligned}
|(\mathbf{u}, p)|_{h}^{2} & =\left\|\nabla_{h} \times \mathbf{u}\right\|_{0, \Omega}^{2}+\alpha\left\|\mathrm{h}^{-\frac{1}{2}} \llbracket \mathbf{u} \rrbracket_{T}\right\|_{0, \mathcal{E}}^{2}+k^{2} \beta\left\|\mathrm{h}^{\frac{1}{2}} \llbracket \mathbf{u} \rrbracket_{N}\right\|_{0, \mathcal{E}_{\mathcal{I}}}^{2} \\
& +k^{2} \beta \sum_{K \in \mathcal{T}_{h}} h_{K}^{2}\|\nabla \cdot \mathbf{u}\|_{0, K}^{2}+k^{2} \gamma\left\|\mathrm{h}^{-\frac{1}{2}} \llbracket p \rrbracket_{N}\right\|_{0, \mathcal{E}}^{2}+\alpha\left\|\mathrm{h}^{-\frac{1}{2}} \llbracket \nabla_{h} p \rrbracket_{T}\right\|_{0, \mathcal{E}}^{2}
\end{aligned}
$$

It is easy to see that $\|(\cdot, \cdot)\|_{h}$ is actually a norm in $\mathbf{V}(h) \times Q(h)$.

Our main result establishes error estimates in the norm $\|(\mathbf{u}, p)\|_{h}$.

Theorem 7 Assume that the exact solution $(\mathbf{u}, p)$ of the continuous problem (3)(6) satisfies

$$
\mathbf{u} \in H^{s}(\Omega)^{3} \quad \nabla \times \mathbf{u} \in H^{s}(\Omega)^{3} \quad p \in H^{s+1}(\Omega) \quad s>\frac{1}{2},
$$

and let $\left(\mathbf{u}_{h}, p_{h}\right)$ be the discrete solution obtained by the DG method (9)-(10). Then, there exists positive constants $\alpha_{0}$ and $\beta_{0}$, with $\alpha_{0}=\alpha_{0}(\kappa, \ell)$ and $\beta_{0}=$ $\beta_{0}\left(\kappa, \ell, C_{\mathrm{ell}}\right)$, such that for $\alpha>\alpha_{0}$ and $\beta>\beta_{0}$ we have the error bound

$$
\left\|\left(\mathbf{u}-\mathbf{u}_{h}, p-p_{h}\right)\right\|_{h} \leq C h^{\min \{\ell, s\}}\left(\|\mathbf{u}\|_{s, \Omega}+\|\nabla \times \mathbf{u}\|_{s, \Omega}+\|p\|_{s+1, \Omega}\right),
$$

provided that $h \leq h_{0}$ for some $h_{0}=h_{0}\left(\kappa, \ell, k, C_{\mathrm{reg}}, \alpha, \beta, \gamma, \Omega, s\right)$.

Remark 8 Theorem 7 guarantees optimal a priori error bounds provided that the stabilization parameters $\alpha$ and $\beta$ are large enough. Restrictions of this type are typically encountered in interior penalty methods (see, e.g., [28,41]). It is worth noting that $\alpha_{0}$ and $\beta_{0}$ are independent of the wave number $k^{2}$.

Remark 9 Note that the smoothness assumptions $\mathbf{u} \in H^{s}(\Omega)^{3}$ and $\nabla \times \mathbf{u} \in$ $H^{s}(\Omega)^{3}$ follow from Proposition 2, whereas the assumption $p \in H^{s+1}(\Omega)$ does not seem to hold true for general source terms $\mathcal{J}$ in $L^{2}(\Omega)^{3}$. This lack of smoothness in the potential p for general right hand sides will be the major difficulty in our duality argument in section 6 below and is the reason why we introduced the stabilization forms $d(\cdot, \cdot)$ and $e(\cdot, \cdot)$. We also point out that for divergence free source terms $\mathcal{J}$ (often encountered in practice) we have $p=0$ and the assumption $p \in H^{s+1}(\Omega)$ is trivially satisfied.

Proceeding along the lines of [42], we can conclude from the a priori error estimates in Theorem 7 existence and uniqueness of discrete solutions.

Corollary 10 For $\alpha>\alpha_{0}$ and $\beta>\beta_{0}$, the DG method (3)-(4) admits a unique solution, provided that $h \leq h_{0}$.

Proof We need only establish that if $\mathcal{J}=\mathbf{0}$, the only solution is $\mathbf{u}_{h}=\mathbf{0}$ and $p_{h}=0$. But if $\mathcal{J}=\mathbf{0}$, then $\mathbf{u}=\mathbf{0}, p=0$ and the estimate of Theorem 7 implies 
$\left\|\left(\mathbf{u}_{h}, p_{h}\right)\right\|_{h} \leq 0$ for $h \leq h_{0}$. Since $\|(\cdot, \cdot)\|_{h}$ is a norm on $\mathbf{V}_{h} \times Q_{h}$, we conclude that $\mathbf{u}_{h}=\mathbf{0}$ and $p_{h}=0$.

The proof of Theorem 7 is developed in section 5 and section 6 . First, we rewrite the DG method in a non-conforming fashion, using lifting operators similar to the ones introduced in [28], and prove an inf-sup condition. Then, we derive an abstract error estimate which can be viewed as a variant of Strang's lemma. This is done in section 5. Finally, in section 6, we make explicit the error estimates for the principal part of the problem and then for the $L^{2}$-norm of the error by a duality approach where we need $\beta$ to be large enough.

\section{Abstract error estimates}

In this section, we prove an abstract error estimate for our DG method. The key ingredient to obtain this estimate is an inf-sup condition which we prove in Proposition 17, following an argument often used in the analysis of stabilized finite elements in Stokes flow (see, e.g., the survey article [43] and the references therein).

\subsection{A global bilinear form}

For the purpose of our analysis, we replace the integrals over interelement boundaries by volume integrals given in terms of the lifting operators $\mathcal{L}: L^{2}(\mathcal{E})^{3} \rightarrow \mathbf{V}_{h}$ and $\mathcal{M}: L^{2}\left(\mathcal{E}_{\mathcal{I}}\right) \rightarrow Q_{h}$ defined by

$$
\begin{aligned}
\left.\int_{\Omega} \mathcal{L}(\mathbf{v}) \cdot \mathbf{w} d \mathbf{x}=\int_{\mathcal{E}} \mathbf{v} \cdot\{\mathbf{w}\}\right] d s & \forall \mathbf{w} \in \mathbf{V}_{h} \\
\int_{\Omega} \mathcal{M}(v) q d \mathbf{x}=\int_{\mathcal{E}_{\mathcal{I}}} v\{\{q\}] d s & \forall q \in Q_{h} .
\end{aligned}
$$

We also need the lifting operator $\mathcal{N}: L^{2}\left(\mathcal{E}_{\mathcal{I}}\right) \rightarrow \mathbf{V}_{h}$ defined by

$$
\int_{\Omega} \mathcal{N}(v) \cdot \mathbf{w} d \mathbf{x}=\int_{\mathcal{E}_{\mathcal{I}}} v \llbracket \mathbf{w} \rrbracket_{N} d s \quad \forall \mathbf{w} \in \mathbf{V}_{h}
$$

Consider the forms $a_{\mathrm{lift}}(\cdot, \cdot)$ and $b_{\mathrm{lift}}(\cdot, \cdot)$ given by

$$
\begin{aligned}
a_{\mathrm{lift}}(\mathbf{u}, \mathbf{v}):= & \int_{\Omega} \nabla_{h} \times \mathbf{u} \cdot \nabla_{h} \times \mathbf{v} d \mathbf{x} \\
& -\int_{\Omega}\left[\mathcal{L}\left(\llbracket \mathbf{u} \rrbracket_{T}\right) \cdot\left(\nabla_{h} \times \mathbf{v}\right)+\mathcal{L}\left(\llbracket \mathbf{v} \rrbracket_{T}\right) \cdot\left(\nabla_{h} \times \mathbf{u}\right)\right] d \mathbf{x} \\
b_{\text {lift }}(\mathbf{v}, p):= & k^{2} \int_{\Omega} p \nabla_{h} \cdot \mathbf{v} d \mathbf{x}-k^{2} \int_{\Omega} \mathcal{M}\left(\llbracket \mathbf{v} \rrbracket_{N}\right) p d \mathbf{x} .
\end{aligned}
$$


Again, by integration by parts, we have

$$
b_{\text {lift }}(\mathbf{v}, p)=-k^{2} \int_{\Omega} \mathbf{v} \cdot \nabla_{h} p d \mathbf{x}+k^{2} \int_{\Omega} \mathcal{L}\left(\llbracket p \rrbracket_{N}\right) \cdot \mathbf{v} d \mathbf{x}, \quad \forall(\mathbf{v}, p) \in \mathbf{V}_{h} \times Q_{h} .
$$

For discrete test and trial functions, the forms $a_{\mathrm{lift}}(\cdot, \cdot)$ and $b_{\mathrm{lift}}(\cdot, \cdot)$ coincide with $a(\cdot, \cdot)$ and $b(\cdot, \cdot)$. However, this is no longer true for continuous functions, due to the discrete nature of the lifting operators. Nevertheless, we carry out our analysis in terms of the forms $a_{\text {lift }}(\cdot, \cdot)$ and $b_{\text {lift }}(\cdot, \cdot)$ since they have more favorable continuity and coercivity properties and take into account the inconsistency of the forms by a variant of Strang's lemma.

Introducing the global form $\mathcal{B}_{h}(\mathbf{u}, p ; \mathbf{v}, q)$ defined by

$$
\begin{aligned}
\mathcal{B}_{h}(\mathbf{u}, p ; \mathbf{v}, q):= & a_{\mathrm{lift}}(\mathbf{u}, \mathbf{v})+c(\mathbf{u}, \mathbf{v})+d(\mathbf{u}, \mathbf{v})-k^{2}(\mathbf{u}, \mathbf{v}) \\
& +b_{\mathrm{lift}}(\mathbf{v}, p)-b_{\mathrm{lift}}(\mathbf{u}, q)+e(p, q)
\end{aligned}
$$

we can rewrite the DG method (9)-(10) in the following compact form:

Find $\left(\mathbf{u}_{h}, p_{h}\right) \in \mathbf{V}_{h} \times Q_{h}$ such that

$$
\mathcal{B}_{h}\left(\mathbf{u}_{h}, p_{h} ; \mathbf{v}, q\right)=\mathcal{F}(\mathbf{v})
$$

for all $(\mathbf{v}, q) \in \mathbf{V}_{h} \times Q_{h}$.

\subsection{Stability of the lifting operators}

The following standard inverse inequalities (see, e.g., [38]) will be useful in the rest of the paper.

Lemma 11 For polynomials $r \in \mathcal{P}^{\ell}(K)$, we have

$$
\|r\|_{0, \partial K} \leq C_{\mathrm{inv}} h_{K}^{-\frac{1}{2}}\|r\|_{0, K} \quad|r|_{1, K} \leq C_{\mathrm{inv}} h_{K}^{-1}\|r\|_{0, K}
$$

with a constant $C_{\mathrm{inv}}>0$ only depending on the shape regularity constant $\kappa$ and the polynomial degree $\ell$.

We start by establishing stability estimates for the lifting operators.

Proposition 12 Let $\mathcal{L}$ and $\mathcal{M}$ be the lifting operators defined above. We have that, for all $\mathbf{v} \in \mathbf{V}(h)$ and for all $q \in Q(h)$,

$$
\begin{array}{ll}
\left\|\mathcal{L}\left(\llbracket \mathbf{v} \rrbracket_{T}\right)\right\|_{0, \Omega} \leq C_{\text {lift }}\left\|\mathrm{h}^{-\frac{1}{2}} \llbracket \mathbf{v} \rrbracket_{T}\right\|_{0, \mathcal{E}} & \left\|\mathcal{L}\left(\llbracket q \rrbracket_{N}\right)\right\|_{0, \Omega} \leq C_{\text {lift }}\left\|\mathrm{h}^{-\frac{1}{2}} \llbracket q \rrbracket_{N}\right\|_{0, \mathcal{E}} \\
\left\|\mathcal{M}\left(\llbracket \mathbf{v} \rrbracket_{N}\right)\right\|_{0, \Omega} \leq C_{\text {lift }}\left\|\mathrm{h}^{-\frac{1}{2}} \llbracket \mathbf{v} \rrbracket_{N}\right\|_{0, \mathcal{E}_{\mathcal{I}}} & \left\|\mathcal{N}\left(\llbracket \mathbf{v} \rrbracket_{N}\right)\right\|_{0, \Omega} \leq C_{\text {lift }}\left\|\mathrm{h}^{-\frac{1}{2}} \llbracket \mathbf{v} \rrbracket_{N}\right\|_{0, \mathcal{E}_{\mathcal{I}}}
\end{array}
$$

with a constant $C_{\text {lift }}$ only depending on $\kappa$ and $\ell$. 
Proof We prove the first estimate. Given $\mathbf{v}=\mathbf{w}_{h}+\mathbf{w} \in \mathbf{V}(h)$, observe that $\llbracket \mathbf{v} \rrbracket_{T}=\llbracket \mathbf{w}_{h} \rrbracket_{T}$ on $\mathcal{E}$. By the definition of the operator $\mathcal{L}$ and the Cauchy-Schwarz inequality, we have

$$
\begin{aligned}
\left\|\mathcal{L}\left(\llbracket \mathbf{v} \rrbracket_{T}\right)\right\|_{0, \Omega} & =\sup _{\mathbf{z} \in \mathbf{V}_{h}} \frac{\int_{\Omega} \mathcal{L}\left(\llbracket \mathbf{v} \rrbracket_{T}\right) \cdot \mathbf{z} d \mathbf{x}}{\|\mathbf{z}\|_{0, \Omega}}=\sup _{\mathbf{z} \in \mathbf{V}_{h}} \frac{\left.\int_{\mathcal{E}} \llbracket \mathbf{v} \rrbracket_{T} \cdot\{\mathbf{z}\}\right\} d s}{\|\mathbf{z}\|_{0, \Omega}} \\
& \leq \sup _{\mathbf{z} \in \mathbf{V}_{h}} \frac{\left\|\mathrm{h}^{-\frac{1}{2}} \llbracket \mathbf{v} \rrbracket_{T}\right\|_{0, \mathcal{E}} \| \mathrm{h}^{\frac{1}{2}}\left\{\{\mathbf{z}\} \|_{0, \mathcal{E}}\right.}{\|\mathbf{z}\|_{0, \Omega}}
\end{aligned}
$$

Then, by using the definitions of $\{[\cdot\}$ and $h$, and the first inverse inequality in Lemma 11, we obtain

$$
\| \mathrm{h}^{\frac{1}{2}}\left\{\{\mathbf{z}\}\left\|_{0, \mathcal{E}}^{2} \leq C \sum_{K \in \mathcal{T}_{h}} h_{K}\right\| \mathbf{z}\left\|_{0, \partial K}^{2} \leq C \sum_{K \in \mathcal{T}_{h}}\right\| \mathbf{z}\left\|_{0, K}^{2}=C\right\| \mathbf{z} \|_{0, \Omega}^{2} .\right.
$$

This proves the first estimate. The other estimates are obtained similarly.

\subsection{Continuity}

We can state the following continuity properties.

Proposition 13 There exists a positive constant $C$ only depending on $\kappa$ and $\ell$ such that, for all $(\mathbf{u}, p),(\mathbf{v}, q) \in \mathbf{V}(h) \times Q(h)$,

$$
\begin{array}{ll}
\left|a_{\text {lift }}(\mathbf{u}, \mathbf{v})\right| \leq C\|(\mathbf{u}, 0)\|_{h}\|(\mathbf{v}, 0)\|_{h} & |c(\mathbf{u}, \mathbf{v})| \leq C\|(\mathbf{u}, 0)\|_{h}\|(\mathbf{v}, 0)\|_{h} \\
\left|b_{\text {lift }}(\mathbf{u}, p)\right| \leq C\|(\mathbf{u}, 0)\|_{h}\|(\mathbf{0}, p)\|_{h} & |d(\mathbf{u}, \mathbf{v})| \leq C\|(\mathbf{u}, 0)\|_{h}\|(\mathbf{v}, 0)\|_{h} \\
& |e(p, q)| \leq C\|(\mathbf{0}, p)\|_{h}\|(\mathbf{0}, q)\|_{h} .
\end{array}
$$

Consequently, for all $(\mathbf{u}, p),(\mathbf{v}, q) \in \mathbf{V}(h) \times Q(h)$,

$$
\mathcal{B}_{h}(\mathbf{u}, p ; \mathbf{v}, q) \leq C_{\text {cont }}\|(\mathbf{u}, p)\|_{h}\|(\mathbf{v}, q)\|_{h},
$$

for a continuity constant $C_{\text {cont }}>0$ only depending on $\kappa$ and $\ell$.

Proof Using the first estimate in Proposition 12, we have

$$
\begin{aligned}
\left|a_{\text {lift }}(\mathbf{u}, \mathbf{v})\right| \leq & \left\|\nabla_{h} \times \mathbf{u}\right\|_{0, \Omega}\left\|\nabla_{h} \times \mathbf{v}\right\|_{0, \Omega}+\left\|\nabla_{h} \times \mathbf{v}\right\|_{0, \Omega}\left\|\mathcal{L}\left(\llbracket \mathbf{u} \rrbracket_{T}\right)\right\|_{0, \Omega} \\
& +\left\|\nabla_{h} \times \mathbf{u}\right\|_{0, \Omega}\left\|\mathcal{L}\left(\llbracket \mathbf{v} \rrbracket_{T}\right)\right\|_{0, \Omega} \leq C\|(\mathbf{u}, 0)\| h\|(\mathbf{v}, 0)\|_{h}
\end{aligned}
$$

Then, owing to the second estimate in Proposition 12,

$$
\left|b_{\text {lift }}(\mathbf{u}, p)\right| \leq k^{2}\left\|\nabla_{h} p-\mathcal{L}\left(\llbracket p \rrbracket_{N}\right)\right\|_{0, \Omega}\|\mathbf{u}\|_{0, \Omega} \leq C\|(\mathbf{u}, 0)\|_{h}\|(0, p)\|_{h} .
$$

The estimates for $c(\cdot, \cdot), d(\cdot, \cdot)$ and $e(\cdot, \cdot)$ are straightforward. 


\subsection{Inf-sup condition}

We show the stability of the form $\mathcal{B}_{h}$ in the following two steps: we start by proving, in Lemma 14, a Gårding inequality for the form $\mathcal{B}_{h}$ in terms of the seminorm $|(\cdot, \cdot)|_{h}$ and then, in Lemma 16 , a stability estimate for $\mathcal{B}_{h}\left(\mathbf{u}, p ;-\nabla_{h} p,-p\right)$; by combining these results, we obtain the inf-sup condition in Proposition 17.

Lemma 14 There exists a positive constant $C$ independent of $h$ and $k$ such that, for all $(\mathbf{u}, p)$ in $\mathbf{V}_{h} \times Q_{h}$,

$$
\mathcal{B}_{h}(\mathbf{u}, p ; \mathbf{u}, p) \geq C|(\mathbf{u}, p)|_{h}^{2}-k^{2}\|\mathbf{u}\|_{0, \Omega}^{2}
$$

provided that $\alpha>C_{\mathrm{lift}}^{2}$, where $C_{\mathrm{lift}}$ is the constant in the estimates of Proposition 12.

Proof First, we prove the following coercivity property: for all $\mathbf{u} \in \mathbf{V}_{h}$,

$$
a_{\mathrm{lift}}(\mathbf{u}, \mathbf{u})+c(\mathbf{u}, \mathbf{u}) \geq C\left(\left\|\nabla_{h} \times \mathbf{u}\right\|_{0, \Omega}^{2}+\alpha\left\|\mathrm{h}^{-\frac{1}{2}} \llbracket \mathbf{u} \rrbracket_{T}\right\|_{0, \mathcal{E}}^{2}\right) .
$$

Using the arithmetic geometric mean inequality $|a b| \leq \frac{\delta}{2 \alpha} a^{2}+\frac{\alpha}{2 \delta} b^{2}$, and the first bound in Proposition 12, we have, for any $\delta>0$,

$$
\begin{aligned}
& a_{\mathrm{lift}}(\mathbf{u}, \mathbf{u})+c(\mathbf{u}, \mathbf{u}) \\
& =\left\|\nabla_{h} \times \mathbf{u}\right\|_{0, \Omega}^{2}-2 \int_{\Omega} \nabla_{h} \times \mathbf{u} \cdot \mathcal{L}\left(\llbracket \mathbf{u} \rrbracket_{T}\right) d \mathbf{x}+\alpha\left\|\mathrm{h}^{-\frac{1}{2}} \llbracket \mathbf{u} \rrbracket_{T}\right\|_{0, \mathcal{E}}^{2} \\
& \geq\left(1-\frac{\delta}{\alpha}\right)\left\|\nabla_{h} \times \mathbf{u}\right\|_{0, \Omega}^{2}-\frac{\alpha}{\delta}\left\|\mathcal{L}\left(\llbracket \mathbf{u} \rrbracket_{T}\right)\right\|_{0, \Omega}^{2}+\alpha\left\|\mathrm{h}^{-\frac{1}{2}} \llbracket \mathbf{u} \rrbracket_{T}\right\|_{0, \mathcal{E}}^{2} \\
& \geq\left(1-\frac{\delta}{\alpha}\right)\left\|\nabla_{h} \times \mathbf{u}\right\|_{0, \Omega}^{2}+\alpha\left(1-\frac{C_{\text {lift }}^{2}}{\delta}\right)\left\|\mathrm{h}^{-\frac{1}{2}} \llbracket \mathbf{u} \rrbracket_{T}\right\|_{0, \mathcal{E}}^{2} .
\end{aligned}
$$

Owing to the assumption $\alpha>C_{\text {lift }}^{2}$, we can take $C_{\text {lift }}^{2}<\delta<\alpha$ and obtain (15). Now, since $\mathcal{B}_{h}(\mathbf{u}, p ; \mathbf{u}, p):=a_{\mathrm{lift}}(\mathbf{u}, \mathbf{u})+c(\mathbf{u}, \mathbf{u})+d(\mathbf{u}, \mathbf{u})-k^{2}\|\mathbf{u}\|_{0, \Omega}^{2}+e(p, p)$, the result immediately follows from the coercivity property (15) and from the definition of the seminorm $|(\cdot, \cdot)|_{h}$.

Remark 15 If we replace the form $a_{\text {lift }}$ by its nonsymmetric variant derived from (11), Lemma 14 holds true for any $\alpha>0$. For the symmetric method in (9)-(10), we will assume throughout the text that $\alpha$ satisfies $\alpha>C_{\mathrm{lift}}^{2}$ (the constant $\alpha_{0}$ in Theorem 7 is actually $C_{\mathrm{lift}}^{2}$ ).

Lemma 16 Let $(\mathbf{u}, p) \in \mathbf{V}_{h} \times Q_{h}$. Then there exist positive constants $C_{1}, C_{2}$ and $C_{3}$ independent of $h$ and $k$ such that

$$
\begin{aligned}
& \left\|\left(\nabla_{h} p, p\right)\right\|_{h} \leq C_{1}\|(\mathbf{u}, p)\|_{h} \\
& \mathcal{B}_{h}\left(\mathbf{u}, p ;-\nabla_{h} p,-p\right) \geq C_{2} k^{2}\left\|\nabla_{h} p\right\|_{0, \Omega}^{2}-C_{3}|(\mathbf{u}, p)|_{h}^{2}-C_{3} k^{2}\|\mathbf{u}\|_{0, \Omega}^{2} .
\end{aligned}
$$


Proof Let us first prove the continuity property. From Lemma 11, the following bounds hold: $\left\|\mathrm{h}^{\frac{1}{2}} \llbracket \nabla_{h} p \rrbracket_{N}\right\|_{0, \mathcal{E}_{\mathcal{I}}} \leq C\left\|\nabla_{h} p\right\|_{0, \Omega}$ and $\|\Delta p\|_{0, K} \leq C h_{K}^{-1}\|\nabla p\|_{0, K}$. Then, from the definition of $\|(\cdot, \cdot)\|_{h}$, we obtain

$$
\begin{aligned}
\left\|\left(\nabla_{h} p, p\right)\right\|_{h}^{2}= & 2 k^{2}\left\|\nabla_{h} p\right\|_{0, \Omega}^{2}+2 \alpha\left\|\mathrm{h}^{-\frac{1}{2}} \llbracket \nabla_{h} p \rrbracket_{T}\right\|_{0, \mathcal{E}}^{2}+k^{2} \beta\left\|\mathrm{h}^{\frac{1}{2}} \llbracket \nabla_{h} p \rrbracket_{N}\right\|_{0, \mathcal{E}_{\mathcal{I}}}^{2} \\
& +k^{2} \beta \sum_{K \in \mathcal{T}_{h}} h_{K}^{2}\|\Delta p\|_{0, K}^{2}+k^{2} \gamma\left\|\mathrm{h}^{-\frac{1}{2}} \llbracket p \rrbracket_{N}\right\|_{0, \mathcal{E}}^{2} \\
\leq & C k^{2}\left\|\nabla_{h} p\right\|_{0, \Omega}^{2}+2 \alpha\left\|\mathrm{h}^{-\frac{1}{2}} \llbracket \nabla_{h} p \rrbracket_{T}\right\|_{0, \mathcal{E}}^{2}+k^{2} \gamma\left\|\mathrm{h}^{-\frac{1}{2}} \llbracket p \rrbracket_{N}\right\|_{0, \mathcal{E}}^{2} \\
\leq & C_{1}\|(\mathbf{u}, p)\|_{h}^{2},
\end{aligned}
$$

for any $\mathbf{u} \in \mathbf{V}_{h}$, with $C_{1}=C_{1}\left(C_{\mathrm{inv}}, \beta\right)$.

In order to prove the bound for $\mathcal{B}_{h}\left(\mathbf{u}, p ;-\nabla_{h} p,-p\right)$, we estimate separately the bilinear forms that are involved. We consider first $a_{\text {lift }}\left(\mathbf{u},-\nabla_{h} p\right)$. From Proposition 12:

$$
\begin{aligned}
a_{\mathrm{lift}}\left(\mathbf{u},-\nabla_{h} p\right) & =\int_{\Omega} \mathcal{L}\left(\llbracket \nabla_{h} p \rrbracket_{T}\right) \cdot \nabla_{h} \times \mathbf{u} d \mathbf{x} \\
& \geq-\frac{C_{\mathrm{lift}}^{2}}{2}\left\|\mathrm{~h}^{-\frac{1}{2}} \llbracket \nabla_{h} p \rrbracket_{T}\right\|_{0, \mathcal{E}}^{2}-\frac{1}{2}\left\|\nabla_{h} \times \mathbf{u}\right\|_{0, \Omega}^{2} .
\end{aligned}
$$

For $c(\cdot, \cdot)$ and $e(\cdot, \cdot)$ we have

$$
\begin{aligned}
& c\left(\mathbf{u},-\nabla_{h} p\right)=-\alpha \int_{\mathcal{E}} \mathrm{h}^{-1} \llbracket \mathbf{u} \rrbracket_{T} \cdot \llbracket \nabla_{h} p \rrbracket_{T} d s \\
& \geq-\frac{\alpha}{2}\left\|\mathrm{~h}^{-\frac{1}{2}} \llbracket \mathbf{u} \rrbracket_{T}\right\|_{0, \mathcal{E}}^{2}-\frac{\alpha}{2}\left\|\mathrm{~h}^{-\frac{1}{2}} \llbracket \nabla_{h} p \rrbracket_{T}\right\|_{0, \mathcal{E}}^{2} \\
& e(p,-p)=-k^{2} \gamma\left\|\mathrm{h}^{-\frac{1}{2}} \llbracket p \rrbracket_{N}\right\|_{0, \mathcal{E}}^{2}-\alpha\left\|\mathrm{h}^{-\frac{1}{2}} \llbracket \nabla_{h} p \rrbracket_{T}\right\|_{0, \mathcal{E}}^{2} \cdot
\end{aligned}
$$

Let us consider now $b_{\text {lift }}(\cdot, \cdot)$. We have again from Proposition 12 with arithmetic geometric mean inequalities

$$
\begin{aligned}
b_{\text {lift }}\left(-\nabla_{h} p, p\right) & =k^{2}\left\|\nabla_{h} p\right\|_{0, \Omega}^{2}-k^{2} \int_{\Omega} \mathcal{L}\left(\llbracket p \rrbracket_{N}\right) \cdot \nabla_{h} p d s \\
& \geq \frac{k^{2}}{2}\left\|\nabla_{h} p\right\|_{0, \Omega}^{2}-\frac{k^{2} C_{\text {lift }}^{2}}{2}\left\|\mathrm{~h}^{-\frac{1}{2}} \llbracket p \rrbracket_{N}\right\|_{0, \mathcal{E}}^{2}
\end{aligned}
$$

and

$$
\begin{aligned}
-b_{\text {lift }}(\mathbf{u},-p) & =-k^{2} \int_{\Omega} \mathbf{u} \cdot \nabla_{h} p d \mathbf{x}+k^{2} \int_{\Omega} \mathcal{L}\left(\llbracket p \rrbracket_{N}\right) \cdot \mathbf{u} d s \\
& \geq-\frac{9}{2} k^{2}\|\mathbf{u}\|_{0, \Omega}^{2}-\frac{k^{2}}{16}\left\|\nabla_{h} p\right\|_{0, \Omega}^{2}-\frac{k^{2} C_{\text {lift }}^{2}}{2}\left\|\mathrm{~h}^{-\frac{1}{2}} \llbracket p \rrbracket_{N}\right\|_{0, \mathcal{E}}^{2}
\end{aligned}
$$


For $d(\cdot, \cdot)$, we have

$$
\begin{aligned}
d\left(\mathbf{u}, \nabla_{h} p\right)= & -k^{2} \beta \int_{\Omega} \mathcal{N}\left(\mathrm{h} \llbracket \mathbf{u} \rrbracket_{N}\right) \cdot \nabla_{h} p d \mathbf{x}-k^{2} \beta \sum_{K \in \mathcal{T}_{h}} h_{K}^{2} \int_{K} \nabla \cdot \mathbf{u} \Delta p d \mathbf{x} \\
\geq & -4 k^{2} \beta^{2} C_{\mathrm{lift}}^{2}\left\|\mathrm{~h}^{\frac{1}{2}} \llbracket \mathbf{u} \rrbracket_{N}\right\|_{0, \mathcal{E}}^{2}-\frac{k^{2}}{16}\left\|\nabla_{h} p\right\|_{0, \Omega}^{2} \\
& -4 k^{2} C^{2} \beta^{2} \sum_{K \in \mathcal{T}_{h}} h_{K}^{2}\|\nabla \cdot \mathbf{u}\|_{0, K}^{2}-\frac{k^{2}}{16}\left\|\nabla_{h} p\right\|_{0, \Omega}^{2},
\end{aligned}
$$

where we used the estimates in Proposition 12, the inverse estimate $\|\Delta p\|_{0, K} \leq$ $C h_{K}^{-1}\|\nabla p\|_{0, K}$ and arithmetic geometric mean inequalities with suitable weights. Finally, we note that

$$
k^{2}\left(\mathbf{u}, \nabla_{h} p\right) \geq-4 k^{2}\|\mathbf{u}\|_{0, \Omega}^{2}-\frac{k^{2}}{16}\left\|\nabla_{h} p\right\|_{0, \Omega}^{2} .
$$

Adding together all the contributions from the bilinear forms we obtain the result (with our choice of the weights, $C_{2}=\frac{1}{4}$ ).

We are now ready to prove the following inf-sup condition.

Proposition 17 There are positive constants $C_{1}, C_{2}$ and $C_{3}$ independent of $h$ and $k$ such that, for any $(\mathbf{v}, q) \in \mathbf{V}_{h} \times Q_{h}$, there is $(\mathbf{w}, s) \in \mathbf{V}_{h} \times Q_{h}$ such that

$$
\begin{aligned}
& \|(\mathbf{w}, s)\|_{h} \leq C_{1}\|(\mathbf{v}, q)\|_{h} \\
& \mathcal{B}_{h}(\mathbf{v}, q ; \mathbf{w}, s) \geq C_{2}\left(|(\mathbf{v}, q)|_{h}^{2}+k^{2}\left\|\nabla_{h} q\right\|_{0, \Omega}^{2}\right)-C_{3} k^{2}\|\mathbf{v}\|_{0, \Omega}^{2} .
\end{aligned}
$$

Proof Set $(\mathbf{w}, s)=\delta(\mathbf{v}, q)-\left(\nabla_{h} q, q\right)$, combine Lemma 14 and Lemma 16 and choose $\delta$ large enough.

\subsection{A variant of Strang's lemma}

We prove the following abstract error estimate involving the residual

$$
\mathcal{R}_{h}(\mathbf{u}, p ; \mathbf{v}, q)=\mathcal{B}_{h}(\mathbf{u}, p ; \mathbf{v}, q)-\mathcal{F}(\mathbf{v})
$$

which takes into account the inconsistency of the formulation (14).

Theorem 18 There is a constant $C$ independent of $h$ and $k$ such that the error $\left(\mathbf{u}-\mathbf{u}_{h}, p-p_{h}\right)$ satisfies

$$
\begin{aligned}
\left\|\left(\mathbf{u}-\mathbf{u}_{h}, p-p_{h}\right)\right\|_{h} \leq & C\left(\inf _{(\mathbf{v}, q) \in \mathbf{V}_{h} \times Q_{h}}\|(\mathbf{u}-\mathbf{v}, p-q)\|_{h}\right. \\
& \left.+\sup _{(\mathbf{0}, 0) \neq(\mathbf{w}, s) \in \mathbf{V}_{h} \times Q_{h}} \frac{\left|\mathcal{R}_{h}(\mathbf{u}, p ; \mathbf{w}, s)\right|}{\|(\mathbf{w}, s)\|_{h}}+k\left\|\mathbf{u}-\mathbf{u}_{h}\right\|_{0, \Omega}\right) .
\end{aligned}
$$


Proof Fix $(\mathbf{v}, q) \in \mathbf{V}_{h} \times Q_{h}$. We split the error $\left(\mathbf{u}-\mathbf{u}_{h}, p-p_{h}\right)$ into

$$
\left(\mathbf{u}-\mathbf{u}_{h}, p-p_{h}\right)=(\mathbf{u}-\mathbf{v}, p-q)+\left(\mathbf{v}-\mathbf{u}_{h}, q-p_{h}\right)=:\left(\boldsymbol{\varphi}_{u}, \varphi_{p}\right)+\left(\boldsymbol{\xi}_{u}, \xi_{p}\right) .
$$

We bound $\left(\boldsymbol{\xi}_{u}, \xi_{p}\right)$, which we may assume to be nonzero. By Proposition 17, there exists a nonzero test function $(\mathbf{w}, s) \in \mathbf{V}_{h} \times Q_{h}$ satisfying (16) with $(\mathbf{v}, q)=$ $\left(\boldsymbol{\xi}_{u}, \xi_{p}\right)$. We obtain

$$
\begin{aligned}
\left\|\left(\boldsymbol{\xi}_{u}, \xi_{p}\right)\right\|_{h}^{2} & =k^{2}\left\|\boldsymbol{\xi}_{u}\right\|_{0, \Omega}^{2}+k^{2}\left\|\nabla_{h} \xi_{p}\right\|_{0, \Omega}^{2}+\left|\left(\boldsymbol{\xi}_{u}, \xi_{p}\right)\right|_{h}^{2} \\
& \leq C\left|\mathcal{B}_{h}\left(\boldsymbol{\xi}_{u}, \xi_{p} ; \mathbf{w}, s\right)\right|+C k^{2}\left\|\boldsymbol{\xi}_{u}\right\|_{0, \Omega}^{2} \\
& \leq C\left|\mathcal{B}_{h}\left(\boldsymbol{\varphi}_{u}, \varphi_{p} ; \mathbf{w}, s\right)\right|+C\left|\mathcal{R}_{h}(\mathbf{u}, p ; \mathbf{w}, s)\right|+C k^{2}\left\|\boldsymbol{\xi}_{u}\right\|_{0, \Omega}^{2} \\
& \leq C \delta^{-1}\left\|\left(\boldsymbol{\varphi}_{u}, \varphi_{p}\right)\right\|_{h}^{2}+C \delta^{-1} \frac{\left|\mathcal{R}_{h}(\mathbf{u}, p ; \mathbf{w}, s)\right|^{2}}{\|(\mathbf{w}, s)\|_{h}^{2}} \\
& +C \delta\|(\mathbf{w}, s)\|_{h}^{2}+C k^{2}\left\|\boldsymbol{\xi}_{u}\right\|_{0, \Omega}^{2} \\
& \leq C \delta^{-1}\left\|\left(\boldsymbol{\varphi}_{u}, \varphi_{p}\right)\right\|_{h}^{2}+C \delta^{-1} \frac{\left|\mathcal{R}_{h}(\mathbf{u}, p ; \mathbf{w}, s)\right|^{2}}{\|(\mathbf{w}, s)\|_{h}^{2}} \\
& +C \delta\left\|\left(\boldsymbol{\xi}_{u}, \xi_{p}\right)\right\|_{h}^{2}+C k^{2}\left\|\boldsymbol{\xi}_{u}\right\|_{0, \Omega}^{2}
\end{aligned}
$$

for any $\delta>0$, where we used the definition of the residual $\mathcal{R}_{h}$, the continuity of $\mathcal{B}_{h}$, arithmetic geometric mean inequalities and $\|(\mathbf{w}, s)\|_{h} \leq C\left\|\left(\boldsymbol{\xi}_{u}, \xi_{p}\right)\right\|_{h}$. Hence, the parameter $\delta$ can be chosen such that

$$
\left\|\left(\boldsymbol{\xi}_{u}, \xi_{p}\right)\right\|_{h}^{2} \leq C\left\|\left(\boldsymbol{\varphi}_{u}, \varphi_{p}\right)\right\|_{h}^{2}+C \frac{\left|\mathcal{R}_{h}(\mathbf{u}, p ; \mathbf{w}, s)\right|^{2}}{\|(\mathbf{w}, s)\|_{h}^{2}}+C k^{2}\left\|\boldsymbol{\xi}_{u}\right\|_{0, \Omega}^{2} .
$$

Since $k^{2}\left\|\boldsymbol{\xi}_{u}\right\|_{0, \Omega}^{2} \leq k^{2}\left\|\mathbf{u}-\mathbf{u}_{h}\right\|_{0, \Omega}^{2}+k^{2}\left\|\boldsymbol{\varphi}_{u}\right\|_{0, \Omega}^{2}$, we have

$$
\begin{aligned}
\left\|\left(\boldsymbol{\xi}_{u}, \xi_{p}\right)\right\|_{h}^{2} \leq C & \left(\left\|\left(\boldsymbol{\varphi}_{u}, \varphi_{p}\right)\right\|_{h}^{2}\right. \\
& \left.+\sup _{(\mathbf{0}, 0) \neq(\mathbf{w}, s) \in \mathbf{V}_{h} \times Q_{h}} \frac{\left|\mathcal{R}_{h}(\mathbf{u}, p ; \mathbf{w}, s)\right|^{2}}{\|(\mathbf{w}, s)\|_{h}^{2}}+k^{2}\left\|\mathbf{u}-\mathbf{u}_{h}\right\|_{0, \Omega}^{2}\right) .
\end{aligned}
$$

The assertion now follows by applying the triangle inequality and taking the infimum over all $(\mathbf{v}, q) \in \mathbf{V}_{h} \times Q_{h}$.

Remark 19 The result of Theorem 18 holds true also in the case $\beta=0$. The positivity of $\beta$ and the stability induced by the corresponding forms will be invoked in the duality argument of the next section.

\section{Error estimates}

In this section, we make explicit the abstract error estimate in Theorem 18. 


\subsection{Approximation properties}

First, we review the approximation results for the $L^{2}$-projection, for standard $H^{1}$ conforming and for curl-conforming Nédéléc operators.

Lemma 20 Let $w \in H^{t}(K), t \geq 0$. Let $\Pi$ be the $L^{2}$-projection from $H^{t}(K)$ onto $\mathcal{P}^{\ell}(K)$. Then for $m$ integer, $0 \leq m \leq t$, we have

$$
|w-\Pi w|_{m, K} \leq C h_{K}^{\min \{\ell+1, t\}-m}\|w\|_{t, K}
$$

Moreover, if $t>\frac{1}{2}$,

$$
\|w-\Pi w\|_{0, \partial K} \leq C h_{K}^{\min \{\ell+1, t\}-\frac{1}{2}}\|w\|_{t, K}
$$

The constant $C$ only depends on $\kappa, \ell$ and $t$.

Proof For natural numbers $t$, the first estimate follows from the classical BrambleHilbert theory (see, e.g., [38]); for $t=0$, it is a consequence of the stability of the $L^{2}$-projection. For non-integer $t$, it can be obtained by interpolation. The second estimate follows from the trace theorem from $L^{2}(\partial K)$ to $H^{t}(K)$, from the first estimate, and scaling arguments.

We also need a standard $H^{1}$-conforming approximant, see [38], and a Clément operator, as constructed in [44].

Lemma 21 The standard nodal $H^{1}$-conforming interpolant $\Pi_{H^{1}}:\left[H^{t+1}(\Omega) \cap\right.$ $\left.H_{0}^{1}(\Omega)\right] \rightarrow\left[Q_{h} \cap H_{0}^{1}(\Omega)\right]$ satisfies

$$
\left\|w-\Pi_{H^{1}} w\right\|_{m, \Omega} \leq C h^{\min \{\ell+1, t+1\}-m}\|w\|_{t+1, \Omega} \quad m=0,1,
$$

for $t>\frac{1}{2}$. The constant $C$ only depends on $\kappa$, $\ell$ and $t$.

This lemma is proved in [38] for integer $t$, and can be proved for non-integer $t$ by using the arguments in [45].

For the Clément operator $\Pi_{\mathrm{Cl}}: H_{0}^{1}(\Omega) \rightarrow\left[Q_{h} \cap H_{0}^{1}(\Omega)\right]$, we recall the following result (see, e.g., [44, pp. 109-111]).

Lemma 22 There exists a constant $C$ only depending on $\kappa$ and $\ell$ such that

$$
\sum_{K \in \mathcal{T}}\left(\left\|w-\Pi_{C l} w\right\|_{1, K}^{2}+h_{K}^{-2}\left\|w-\Pi_{C l} w\right\|_{0, K}^{2}+h_{K}^{-1}\left\|w-\Pi_{C l} w\right\|_{0, \partial K}^{2}\right) \leq C\|w\|_{1, \Omega}^{2}
$$

Finally, we establish the next lemma for curl-conforming Nédéléc operators. 
Lemma 23 Let $\Pi_{\text {curl }}$ be the curl-conforming Nédéléc operator (either of the first type [6] or of the second type [7]) into $\mathrm{V}_{h} \cap H_{0}(\operatorname{curl} ; \Omega)$. Then, there exists a constant $C=C(\kappa, \ell, t)$ such that, for any $\mathbf{w} \in H_{0}(\operatorname{curl} ; \Omega) \cap H^{t}(\Omega)^{3}$ with $\nabla \times \mathbf{w} \in$ $H^{t}(\Omega)^{3}, t>\frac{1}{2}$,

$$
\left\|\mathbf{w}-\Pi_{\text {curl }} \mathbf{w}\right\|_{\text {curl }, \Omega} \leq C h^{\min \{\ell, t\}}\left(\|\mathbf{w}\|_{t, \Omega}+\|\nabla \times \mathbf{w}\|_{t, \Omega}\right) .
$$

Moreover, if $\mathbf{w}$ also belongs to $H\left(\operatorname{div}^{0} ; \Omega\right)$,

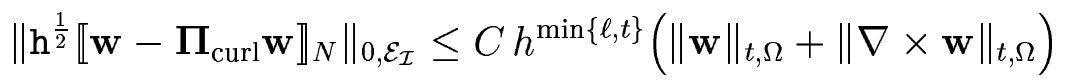

$$
\begin{aligned}
& \left(\sum_{K \in \mathcal{T}_{h}} h_{K}^{2}\left\|\nabla \cdot\left(\mathbf{w}-\Pi_{\text {curl }} \mathbf{w}\right)\right\|_{0, K}^{2}\right)^{\frac{1}{2}} \leq C h^{\min \{\ell, t\}}\left(\|\mathbf{w}\|_{t, \Omega}+\|\nabla \times \mathbf{w}\|_{t, \Omega}\right) \text {. }
\end{aligned}
$$

Proof The first part is proved in [45, Section 5]. For the second part, denote by $\Pi_{\mathbf{V}_{h}}$ the $L^{2}$-projection onto $\mathbf{V}_{h}$. By using the triangle inequality, the approximation results in Lemma 20, the first inverse estimate in Lemma 11, the $L^{2}$-stability of $\Pi_{\mathbf{V}_{h}}$ and the first part of this lemma, we obtain

$$
\begin{aligned}
\| \mathrm{h}^{\frac{1}{2}} \llbracket \mathbf{w} & -\Pi_{\text {curl }} \mathbf{w} \rrbracket_{N} \|_{0, \mathcal{E}_{\mathcal{I}}}^{2} \\
& \leq C \sum_{K \in \mathcal{T}_{h}} h_{K}\left(\left\|\mathbf{w}-\Pi_{\mathbf{V}_{h}} \mathbf{w}\right\|_{0, \partial K}^{2}+\left\|\Pi_{\mathbf{V}_{h}}\left(\mathbf{w}-\Pi_{\text {curl }} \mathbf{w}\right)\right\|_{0, \partial K}^{2}\right) \\
& \leq C \sum_{K \in \mathcal{T}_{h}} h_{K}^{2 \min \{\ell+1, t\}}\|\mathbf{w}\|_{t, K}^{2}+C \sum_{K \in \mathcal{T}_{h}}\left\|\Pi_{\mathbf{V}_{h}}\left(\mathbf{w}-\Pi_{\text {curl } \mathbf{w}}\right)\right\|_{0, K}^{2} \\
& \leq C h^{2 \min \{\ell+1, t\}}\|\mathbf{w}\|_{t, \Omega}^{2}+C\left\|\mathbf{w}-\Pi_{\text {curl }} \mathbf{w}\right\|_{0, \Omega}^{2} \\
& \leq C h^{2 \min \{\ell, t\}}\left(\|\mathbf{w}\|_{t, \Omega}+\|\nabla \times \mathbf{w}\|_{t, \Omega}\right)^{2} .
\end{aligned}
$$

To prove the last estimate, we integrate by parts and obtain

$$
\begin{aligned}
& \sum_{K \in \mathcal{T}_{h}} h_{K}^{2}\left\|\nabla \cdot\left(\mathbf{w}-\Pi_{\mathrm{curl}} \mathbf{w}\right)\right\|_{0, K}^{2} \\
= & -\sum_{K \in \mathcal{T}_{h}} h_{K}^{2} \int_{K} \nabla \nabla \cdot\left(\mathbf{w}-\Pi_{\mathrm{curl}} \mathbf{w}\right) \cdot\left(\mathbf{w}-\Pi_{\mathrm{curl}} \mathbf{w}\right) d \mathbf{x} \\
& +\sum_{K \in \mathcal{T}_{h}} h_{K}^{2} \int_{\partial K} \nabla \cdot\left(\mathbf{w}-\Pi_{\mathrm{curl}} \mathbf{w}\right)\left(\mathbf{w}-\Pi_{\mathrm{curl}} \mathbf{w}\right) \cdot \mathbf{n}_{K} d s=: T_{1}+T_{2} .
\end{aligned}
$$

Let us first consider the volume term $T_{1}$. Using the fact that $\nabla \cdot \mathbf{w}=0$, CauchySchwarz inequalities, the second inverse estimate in Lemma 11 and the first part of 
this lemma, we get

$$
\begin{aligned}
T_{1} & \leq\left(\sum_{K \in \mathcal{T}_{h}} h_{K}^{4}\left\|\nabla \nabla \cdot \Pi_{\mathrm{curl}} \mathbf{w}\right\|_{0, K}^{2}\right)^{\frac{1}{2}}\left\|\mathbf{w}-\Pi_{\mathrm{curl}} \mathbf{w}\right\|_{0, \Omega} \\
& \leq C\left(\sum_{K \in \mathcal{T}_{h}} h_{K}^{2}\left\|\nabla \cdot \boldsymbol{\Pi}_{\mathrm{curl}} \mathbf{w}\right\|_{0, K}^{2}\right)^{\frac{1}{2}}\left\|\mathbf{w}-\boldsymbol{\Pi}_{\mathrm{curl}} \mathbf{w}\right\|_{0, \Omega} \\
& =C\left(\sum_{K \in \mathcal{T}_{h}} h_{K}^{2}\left\|\nabla \cdot\left(\mathbf{w}-\boldsymbol{\Pi}_{\mathrm{curl}} \mathbf{w}\right)\right\|_{0, K}^{2}\right)^{\frac{1}{2}}\left\|\mathbf{w}-\boldsymbol{\Pi}_{\mathrm{curl}} \mathbf{w}\right\|_{0, \Omega} \\
& \leq C h^{\min \{\ell, t\}}\left(\sum_{K \in \mathcal{T}_{h}} h_{K}^{2}\left\|\nabla \cdot\left(\mathbf{w}-\Pi_{\mathrm{curl}} \mathbf{w}\right)\right\|_{0, K}^{2}\right)^{\frac{1}{2}}\left(\|\mathbf{w}\|_{t, \Omega}+\|\nabla \times \mathbf{w}\|_{t, \Omega}\right) .
\end{aligned}
$$

Similarly, we can bound the term $T_{2}$ by

$$
\begin{aligned}
T_{2} \leq & \left(\sum_{K \in \mathcal{T}_{h}} h_{K}^{3}\left\|\nabla \cdot \Pi_{\text {curl }} \mathbf{w}\right\|_{0, \partial K}^{2}\right)^{\frac{1}{2}}\left(\sum_{K \in \mathcal{T}_{h}} h_{K}\left\|\mathbf{w}-\Pi_{\text {curl }} \mathbf{w}\right\|_{0, \partial K}^{2}\right)^{\frac{1}{2}} \\
\leq & \left(\sum_{K \in \mathcal{T}_{h}} h_{K}^{2}\left\|\nabla \cdot \Pi_{\mathrm{curl}} \mathbf{w}\right\|_{0, K}^{2}\right)^{\frac{1}{2}} \\
& \left(\sum_{K \in \mathcal{T}_{h}} h_{K}\left\|\mathbf{w}-\Pi_{\mathbf{V}_{h}} \mathbf{w}\right\|_{0, \partial K}^{2}+\left\|\Pi_{\mathbf{V}_{h}}\left(\mathbf{w}-\Pi_{\text {curl }} \mathbf{w}\right)\right\|_{0, \partial K}^{2}\right)^{\frac{1}{2}} \\
& \leq C h^{\min \{\ell, t\}}\left(\sum_{K \in \mathcal{T}_{h}} h_{K}^{2}\left\|\nabla \cdot\left(\mathbf{w}-\Pi_{\text {curl }} \mathbf{w}\right)\right\|_{0, K}^{2}\right)^{\frac{1}{2}}\left(\|\mathbf{w}\|_{t, \Omega}+\|\nabla \times \mathbf{w}\|_{t, \Omega}\right),
\end{aligned}
$$

where we used the first inverse estimate in Lemma 11 for the term containing the sum and proceeded as in the estimate of $\left\|\mathrm{h}^{\frac{1}{2}} \llbracket \mathbf{w}-\Pi_{\text {curl }} \mathbf{w} \rrbracket_{N}\right\|_{0, \mathcal{E}_{\mathcal{I}}}$ for the second term. This completes the proof of the last estimate.

\subsection{Error in the principal part}

We have the following estimate of the residual in Theorem 18.

Lemma 24 Let $(\mathbf{u}, p)$ be the exact solution and assume that $\nabla \times \mathbf{u} \in H^{s}(\Omega)^{3}$ and $p \in H^{s+1}(\Omega)$, for $s>\frac{1}{2}$. Then, for all $(\mathbf{v}, q) \in \mathbf{V}(h) \times Q(h)$,

$$
\begin{aligned}
\mathcal{R}_{h}(\mathbf{u}, p ; \mathbf{v}, q)= & \int_{\mathcal{E}} \llbracket \mathbf{v} \rrbracket_{T} \cdot\left\{\left\{\nabla \times \mathbf{u}-\Pi_{\mathbf{V}_{h}}(\nabla \times \mathbf{u})\right\} d s\right. \\
& +k^{2} \int_{\mathcal{E}_{\mathcal{I}}} \llbracket \mathbf{v} \rrbracket_{N}\left\{\left\{p-\Pi_{Q_{h}} p\right\}\right\} d s,
\end{aligned}
$$

where $\Pi_{\mathbf{V}_{h}}$ and $\Pi_{Q_{h}}$ denote the $L^{2}$-projections onto $\mathbf{V}_{h}$ and $Q_{h}$, respectively. Moreover, there exists $C$ independent of $h$ such that

$$
\left|\mathcal{R}_{h}(\mathbf{u}, p ; \mathbf{v}, q)\right| \leq C h^{\min \{\ell, s\}}\|(\mathbf{v}, 0)\|_{h}\left(\|\nabla \times \mathbf{u}\|_{s, \Omega}^{2}+\|p\|_{s+1, \Omega}^{2}\right)^{\frac{1}{2}}
$$


Proof By straightforward calculations involving integration by parts and taking into account the definition of the lifting operators $\mathcal{L}$ and $\mathcal{M}$, we have that, for any $(\mathbf{v}, p) \in \mathbf{V}(h) \times Q(h)$,

$$
\begin{aligned}
\mathcal{R}_{h}(\mathbf{u}, p ; \mathbf{v}, q)= & \int_{\mathcal{E}}\left\{\langle\nabla \times \mathbf{u}\} \cdot \llbracket \mathbf{v} \rrbracket_{T} d s-\int_{\Omega} \nabla \times \mathbf{u} \cdot \mathcal{L}\left(\llbracket \mathbf{v} \rrbracket_{T}\right) d \mathbf{x}\right. \\
& +k^{2} \int_{\mathcal{E}_{\mathcal{I}}}\{\{p\}\} \llbracket \mathbf{v} \rrbracket_{N} d s-k^{2} \int_{\Omega} p \mathcal{M}\left(\llbracket \mathbf{v} \rrbracket_{N}\right) d \mathbf{x} .
\end{aligned}
$$

Since

$$
\begin{aligned}
& \int_{\Omega} \nabla \times \mathbf{u} \cdot \mathcal{L}\left(\llbracket \mathbf{v} \rrbracket_{T}\right) d \mathbf{x}=\int_{\Omega} \Pi_{\mathbf{V}_{h}}(\nabla \times \mathbf{u}) \cdot \mathcal{L}\left(\llbracket \mathbf{v} \rrbracket_{T}\right) d \mathbf{x} \\
& \int_{\Omega} p \mathcal{M}\left(\llbracket \mathbf{v} \rrbracket_{N}\right) d \mathbf{x}=\int_{\Omega} \Pi_{Q_{h}} p \mathcal{M}\left(\llbracket \mathbf{v} \rrbracket_{N}\right) d \mathbf{x},
\end{aligned}
$$

we obtain the desired expression for $\mathcal{R}_{h}(\mathbf{u}, p ; \mathbf{v}, q)$.

For the estimates of the residual, let us write $\mathcal{R}_{h}(\mathbf{u}, p ; \mathbf{v}, q)=: T_{1}+T_{2}$, where

$$
\begin{aligned}
T_{1} & =\int_{\mathcal{E}} \llbracket \mathbf{v} \rrbracket_{T} \cdot\left\{\left[\nabla \times \mathbf{u}-\Pi_{\mathbf{V}_{h}}(\nabla \times \mathbf{v})\right\} d s\right. \\
T_{2} & =k^{2} \int_{\mathcal{E}_{\mathcal{I}}} \llbracket \mathbf{v} \rrbracket_{N}\left\{\left\{p-\Pi_{Q_{h}} p\right\}\right\} d s .
\end{aligned}
$$

By the Cauchy-Schwarz inequality, the definition of the norm $\|(\cdot, \cdot)\|_{h}$ and the second estimate of Lemma 20, we obtain the following bound:

$$
\begin{aligned}
T_{1} & \leq C\|(\mathbf{v}, 0)\|_{h}\left(\sum_{K \in \mathcal{T}_{h}} h_{K}\left\|\nabla \times \mathbf{u}-\Pi_{\mathbf{V}_{h}}(\nabla \times \mathbf{u})\right\|_{0, \partial K}^{2}\right)^{\frac{1}{2}} \\
& \leq C\|(\mathbf{v}, 0)\|_{h}\left(\sum_{K \in \mathcal{T}_{h}} h_{K}^{2 \min \{\ell+1, s\}}\|\nabla \times \mathbf{u}\|_{s, K}^{2}\right)^{\frac{1}{2}}
\end{aligned}
$$

Similarly,

$$
\begin{aligned}
T_{2} & \leq C\|(\mathbf{v}, 0)\|_{h}\left(\sum_{K \in \mathcal{T}_{h}} h_{K}^{-1}\left\|p-\Pi_{Q_{h}} p\right\|_{0, \partial K}^{2}\right)^{\frac{1}{2}} \\
& \leq C\|(\mathbf{v}, 0)\|_{h}\left(\sum_{K \in \mathcal{T}_{h}} h_{K}^{2 \min \{\ell, s\}}\|p\|_{s+1, K}^{2}\right)^{\frac{1}{2}} .
\end{aligned}
$$

The estimate for $\mathcal{R}_{h}$ then follows.

We are now ready to prove the following error estimate.

Corollary 25 Under the assumptions of Theorem 7, there exists a constant $C$ independent of the meshsize $h$ such that

$\left\|\left(\mathbf{u}-\mathbf{u}_{h}, p-p_{h}\right)\right\|_{h} \leq C h^{\min \{\ell, s\}}\left(\|\mathbf{u}\|_{s, \Omega}+\|\nabla \times \mathbf{u}\|_{s, \Omega}+\|p\|_{s+1, \Omega}\right)+C k\left\|\mathbf{u}-\mathbf{u}_{h}\right\|_{0, \Omega}$. 
Proof Consider the abstract estimate of Theorem 18 and bound the infimum by $\left\|\left(\mathbf{u}-\Pi_{\text {curl }} \mathbf{u}, p-\Pi_{H^{1}} p\right)\right\|_{h}$, where $\Pi_{\text {curl }}$ is the curl-conforming Nédéléc operator and $\Pi_{H^{1}}$ the standard $H^{1}$-conforming interpolant from Lemma 21. Taking into account the approximation properties in Lemma 21 and Lemma 23 and the estimate of the residual in Lemma 24, we obtain the result.

\subsection{Error in the $L^{2}$-norm}

In order to complete our error analysis, we need to estimate the term $k\left\|\mathbf{u}-\mathbf{u}_{h}\right\|_{0, \Omega}$. This is done in the next proposition by a duality approach. The main difficulty in this argument is that we can not assume any smoothness for the scalar potential of the dual solution. To overcome these difficulties we will have to make the stabilization constant $\beta$ large enough.

Proposition 26 Let $\sigma>\frac{1}{2}$ be the regularity exponent from Proposition 2. Furthermore, we assume that the exact solution satisfies the smoothness assumptions in (13) with $s>\frac{1}{2}$. Then we have

$$
\begin{aligned}
k\left\|\mathbf{u}-\mathbf{u}_{h}\right\|_{0, \Omega} \leq & C_{1} h^{\min \{\sigma, 1\}}\left\|\left(\mathbf{u}-\mathbf{u}_{h}, p-p_{h}\right)\right\|_{h}+C_{1} h^{\min \{\ell, s\}}\|p\|_{s+1, \Omega} \\
& +C_{2} \beta^{-\frac{1}{2}}\left\|\left(\mathbf{u}-\mathbf{u}_{h}, p-p_{h}\right)\right\|_{h},
\end{aligned}
$$

with $C_{1}=C_{1}\left(\kappa, \ell, k, \beta, C_{\mathrm{reg}}, \sigma, s\right)$ and $C_{2}=C_{2}\left(\kappa, \ell, C_{\mathrm{ell}}\right)$.

Consequently, there exists $\beta_{0}=\beta_{0}\left(\kappa, \ell, C_{\mathrm{ell}}\right)$ such that for $\beta>\beta_{0}$

$$
k\left\|\mathbf{u}-\mathbf{u}_{h}\right\|_{0, \Omega} \leq \frac{1}{2}\left\|\left(\mathbf{u}-\mathbf{u}_{h}, p-p_{h}\right)\right\|_{h}+C h^{\min \{\ell, s\}}\|p\|_{s+1, \Omega},
$$

provided that $h \leq h_{0}$ for a constant $h_{0}=h_{0}\left(\kappa, \ell, k, C_{\mathrm{reg}}, \alpha, \beta, \gamma, \sigma, s\right)$.

Proof The proof is given in several steps. We start by introducing a suitable adjoint problem with right-hand side $k^{2}\left(\mathbf{u}-\mathbf{u}_{h}\right)$, denoting by $(\mathbf{z}, \psi)$ its solution, and we express $k^{2}\left\|\mathbf{u}-\mathbf{u}_{h}\right\|_{0, \Omega}^{2}$ as the sum of $\mathcal{B}_{h}\left(\mathbf{u}-\mathbf{u}_{h}, p-p_{h} ; \mathbf{z}-\boldsymbol{\Pi}_{\text {curl }} \mathbf{z}, \psi-\Pi_{\mathrm{Cl}} \psi\right)$ plus residual terms, with $\boldsymbol{\Pi}_{\text {curl }}$ the curl-conforming Nédéléc operator into $\mathbf{V}_{h} \cap$ $H_{0}(\operatorname{curl} ; \Omega)$ and $\Pi_{\mathrm{Cl}}$ the Clément operator into $Q_{h} \cap H_{0}^{1}(\Omega)$ (step 1). Then, estimates of the residuals (step 2) and of the bilinear forms in the definition of $\mathcal{B}_{h}$ give the result (steps 3 and 4).

Step 1: A dual problem. Let $(\mathbf{z}, \psi)$ be the solution of the dual problem

$$
\begin{aligned}
\nabla \times \nabla \times \mathbf{z}-k^{2} \mathbf{z}+k^{2} \nabla \psi & =k^{2}\left(\mathbf{u}-\mathbf{u}_{h}\right) & & \text { in } \Omega \\
\nabla \cdot \mathbf{z} & =0 & & \text { in } \Omega \\
\mathbf{n} \times \mathbf{z} & =\mathbf{0} & & \text { on } \partial \Omega \\
\psi & =0 & & \text { on } \partial \Omega .
\end{aligned}
$$


By Proposition 1 and Proposition 2, we have

$$
\begin{aligned}
\|\mathbf{z}\|_{\sigma, \Omega}+\|\nabla \times \mathbf{z}\|_{\sigma, \Omega} & \leq C_{\mathrm{reg}} k^{2}\left\|\mathbf{u}-\mathbf{u}_{h}\right\|_{0, \Omega} \\
\|\psi\|_{1, \Omega} & \leq C_{\mathrm{ell}}\left\|\mathbf{u}-\mathbf{u}_{h}\right\|_{0, \Omega}
\end{aligned}
$$

for a regularity exponent $\sigma=\sigma(\Omega)>\frac{1}{2}$. We may also assume that $\sigma \leq 1$. The main problem in the following arguments is that we can not assume $\psi$ to be smoother than belonging to $H_{0}^{1}(\Omega)$.

The solution $(\mathbf{z}, \psi)$ of problem (19) satisfies

$$
\mathcal{B}_{h}(\mathbf{z},-\psi ; \mathbf{v}, q)-\mathcal{R}_{h}(\mathbf{z},-\psi ; \mathbf{v}, q)=k^{2}\left(\mathbf{u}-\mathbf{u}_{h}, \mathbf{v}\right)
$$

for all $(\mathbf{v}, q) \in H^{s}\left(\mathcal{T}_{h}\right)^{3} \times H^{s}\left(\mathcal{T}_{h}\right), s>\frac{1}{2}$. Taking $(\mathbf{v}, q)=\left(\mathbf{u}-\mathbf{u}_{h},-\left(p-p_{h}\right)\right)$, observing (13), we obtain

$\mathcal{B}_{h}\left(\mathbf{z},-\psi ; \mathbf{u}-\mathbf{u}_{h},-\left(p-p_{h}\right)\right)-\mathcal{R}_{h}\left(\mathbf{z},-\psi ; \mathbf{u}-\mathbf{u}_{h},-\left(p-p_{h}\right)\right)=k^{2}\left\|\mathbf{u}-\mathbf{u}_{h}\right\|_{0, \Omega}^{2}$.

Since $\mathcal{B}_{h}\left(\mathbf{u}-\mathbf{u}_{h}, p-p_{h} ; \mathbf{z}_{h}, \psi_{h}\right)=\mathcal{R}_{h}\left(\mathbf{u}, p ; \mathbf{z}_{h}, \psi_{h}\right)=-\mathcal{R}_{h}\left(\mathbf{u}, p ; \mathbf{z}-\mathbf{z}_{h}, \psi-\psi_{h}\right)$, for all $\left(\mathbf{z}_{h}, \psi_{h}\right) \in \mathbf{V}_{h} \times Q_{h}$, and using the skew-symmetry properties of $\mathcal{B}_{h}$, we can write

$$
k^{2}\left\|\mathbf{u}-\mathbf{u}_{h}\right\|_{0, \Omega}^{2}=\mathcal{B}_{h}\left(\mathbf{u}-\mathbf{u}_{h}, p-p_{h} ; \mathbf{z}-\mathbf{z}_{h}, \psi-\psi_{h}\right)+R_{1}+R_{2}+R_{3}+R_{4}
$$

with residual terms

$$
\begin{aligned}
& R_{1}=-\int_{\mathcal{E}} \llbracket \mathbf{u}-\mathbf{u}_{h} \rrbracket_{T} \cdot\left\{\left\{\nabla \times \mathbf{z}-\Pi_{\mathbf{V}_{h}}(\nabla \times \mathbf{z})\right\} d s,\right. \\
& R_{2}=k^{2} \int_{\mathcal{E}_{\mathcal{I}}} \llbracket \mathbf{u}-\mathbf{u}_{h} \rrbracket_{N}\left\{\left\{\psi-\Pi_{Q_{h}} \psi\right\}\right\} d s, \\
& R_{3}=-\int_{\mathcal{E}} \llbracket \mathbf{z}-\mathbf{z}_{h} \rrbracket_{T} \cdot\left\{\left\{\nabla \times \mathbf{u}-\Pi_{\mathbf{V}_{h}}(\nabla \times \mathbf{u})\right\} d s,\right. \\
& R_{4}=-k^{2} \int_{\mathcal{E}_{\mathcal{I}}} \llbracket \mathbf{z}-\mathbf{z}_{h} \rrbracket_{N}\left\{\left\{p-\Pi_{Q_{h}} p\right\}\right\} d s .
\end{aligned}
$$

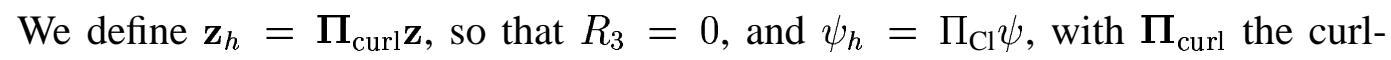
conforming Nédéléc operator from Lemma 23 and $\Pi_{\mathrm{Cl}}$ the standard Clément operator which satisfy the approximation property of Lemma 22.

Step 2: The residuals. We estimate the residual expressions $R_{1}, R_{2}$ and $R_{4}$ in (21) (recall that $R_{3}=0$ ). Let us start with $R_{1}$. The Cauchy-Schwarz inequality and the approximation properties in Lemma 20 yield

$$
\begin{aligned}
R_{1} & \leq C\left\|\left(\mathbf{u}-\mathbf{u}_{h}, 0\right)\right\|_{h}\left(\sum_{K \in \mathcal{T}_{h}} h_{K}\left\|\nabla \times \mathbf{z}-\Pi_{\mathbf{V}_{h}}(\nabla \times \mathbf{z})\right\|_{0, \partial K}^{2}\right)^{\frac{1}{2}} \\
& \leq C h^{\sigma}\left\|\left(\mathbf{u}-\mathbf{u}_{h}, 0\right)\right\| h\|\nabla \times \mathbf{z}\|_{\sigma, \Omega} .
\end{aligned}
$$

Thus, from (20),

$$
R_{1} \leq C h^{\sigma} k^{2}\left\|\left(\mathbf{u}-\mathbf{u}_{h}, 0\right)\right\|_{h}\left\|\mathbf{u}-\mathbf{u}_{h}\right\|_{0, \Omega}
$$


The crucial term is $R_{2}$. Again from the Cauchy-Schwarz inequality, using the second estimate of Lemma 20 and the stability estimates (20) for the dual solution, we obtain

$$
\begin{aligned}
R_{2} & \leq C\left(k^{2} \beta\left\|h^{\frac{1}{2}} \llbracket \mathbf{u}-\mathbf{u}_{h} \rrbracket_{N}\right\|_{0, \mathcal{E}_{\mathcal{I}}}\right)^{\frac{1}{2}}\left(k^{2} \beta^{-1} \sum_{K \in \mathcal{T}_{h}} h_{K}^{-1}\left\|\psi-\Pi_{Q_{h}} \psi\right\|_{0, \partial K}^{2}\right)^{\frac{1}{2}} \\
& \leq C k \beta^{-\frac{1}{2}}\left\|\left(\mathbf{u}-\mathbf{u}_{h}, 0\right)\right\|_{h}\|\psi\|_{1, \Omega} \\
& \leq C k \beta^{-\frac{1}{2}}\left\|\left(\mathbf{u}-\mathbf{u}_{h}, 0\right)\right\|_{h}\left\|\mathbf{u}-\mathbf{u}_{h}\right\|_{0, \Omega},
\end{aligned}
$$

with $C=C\left(\kappa, \ell, C_{\text {ell }}\right)$. Finally, for $R_{4}$ we have

$$
\begin{aligned}
R_{4} & \leq C\left\|\left(\mathbf{z}-\Pi_{\text {curl }} \mathbf{z}, 0\right)\right\|_{h}\left(k^{2} \beta^{-1} \sum_{K \in \mathcal{T}_{h}} h_{K}^{-1}\left\|p-\Pi_{Q_{h}} p\right\|_{0, \partial K}^{2}\right)^{\frac{1}{2}} \\
& \leq C h^{\min \{\ell, s\}} k\left\|\left(\mathbf{z}-\Pi_{\text {curl }} \mathbf{z}, 0\right)\right\|\left\|_{h}\right\| p \|_{s+1, \Omega} .
\end{aligned}
$$

Step 3: The term $\left\|\left(\mathbf{z}-\Pi_{\text {curl }} \mathbf{z}, 0\right)\right\|_{h}$. We claim that

$$
\left\|\left(\mathbf{z}-\Pi_{\mathrm{curl}} \mathbf{z}, 0\right)\right\|_{h} \leq C h^{\sigma} k\left\|\mathbf{u}-\mathbf{u}_{h}\right\|_{0, \Omega},
$$

with $C=C\left(\kappa, \ell, k, C_{\mathrm{reg}}, \beta, \sigma\right)$.

To see (22), we first note that, since the Nédéléc projection is curl-conforming, $\left\|\mathrm{h}^{-\frac{1}{2}} \llbracket \mathbf{z}-\Pi_{\text {curl }} \mathbf{z} \rrbracket_{T}\right\|_{0, \mathcal{E}}=0$. Furthermore, by (17) and (20),

$$
\begin{aligned}
\left\|\nabla \times\left(\mathbf{z}-\Pi_{\mathrm{curl}} \mathbf{z}\right)\right\|_{0, \Omega}^{2}+k^{2}\left\|\mathbf{z}-\Pi_{\mathrm{curl}} \mathbf{z}\right\|_{0, \Omega}^{2} & \leq C h^{2 \sigma}\left(\|\mathbf{z}\|_{\sigma, \Omega}+\|\nabla \times \mathbf{z}\|_{\sigma, \Omega}\right)^{2} \\
& \leq C h^{2 \sigma} k^{4}\left\|\mathbf{u}-\mathbf{u}_{h}\right\|_{0, \Omega}^{2} .
\end{aligned}
$$

From the second and third estimates of Lemma 23 and (20), we have

$$
\begin{aligned}
& k^{2} \beta\left\|\mathrm{h}^{\frac{1}{2}} \llbracket \mathbf{z}-\boldsymbol{\Pi}_{\mathrm{curl}} \mathbf{z} \rrbracket_{N}\right\|_{0, \mathcal{E}_{\mathcal{I}}}^{2} \leq C h^{2 \sigma} k^{2}\left\|\mathbf{u}-\mathbf{u}_{h}\right\|_{0, \Omega}^{2} \\
& k^{2} \beta \sum_{K \in \mathcal{T}} h_{K}^{2}\left\|\nabla \cdot\left(\mathbf{z}-\boldsymbol{\Pi}_{\text {curl }} \mathbf{z}\right)\right\|_{0, K}^{2} \leq C h^{2 \sigma} k^{2}\left\|\mathbf{u}-\mathbf{u}_{h}\right\|_{0, \Omega}^{2}
\end{aligned}
$$

The proof of estimate (22) now follows from the definition of $\left\|\left(\mathbf{z}-\boldsymbol{\Pi}_{\text {curl }} \mathbf{z}, 0\right)\right\|_{h}$.

Step 4: The assertion. We are now able to complete the proof Proposition 26. Define $\boldsymbol{\xi}_{z}=\mathbf{z}-\boldsymbol{\Pi}_{\text {curl }} \mathbf{z}$ and $\xi_{\psi}=\psi-\Pi_{\mathrm{Cl}} \psi$. From (21), taking into account that, due to the conformity of the projectors $\Pi_{\text {curl }}$ and $\Pi_{\mathrm{Cl}}, c\left(\mathbf{u}-\mathbf{u}_{h}, \boldsymbol{\xi}_{z}\right)=0$ and $e\left(p-p_{h}, \xi_{\psi}\right)=0$, we have that

$$
\begin{aligned}
k^{2}\left\|\mathbf{u}-\mathbf{u}_{h}\right\|_{0, \Omega}^{2}= & a_{\text {lift }}\left(\mathbf{u}-\mathbf{u}_{h}, \boldsymbol{\xi}_{z}\right)+d\left(\mathbf{u}-\mathbf{u}_{h}, \boldsymbol{\xi}_{z}\right)-k^{2}\left(\mathbf{u}-\mathbf{u}_{h}, \boldsymbol{\xi}_{z}\right) \\
& +b_{\text {lift }}\left(\boldsymbol{\xi}_{z}, p-p_{h}\right)-b_{\text {lift }}\left(\mathbf{u}-\mathbf{u}_{h}, \xi_{\psi}\right)+R_{1}+R_{2}+R_{3} .
\end{aligned}
$$

From the continuity properties of Proposition 13, we obtain

$$
\begin{aligned}
k^{2}\left\|\mathbf{u}-\mathbf{u}_{h}\right\|_{0, \Omega}^{2} \leq & C\left\|\left(\mathbf{u}-\mathbf{u}_{h}, p-p_{h}\right)\right\|_{h}\left\|\left(\boldsymbol{\xi}_{z}, 0\right)\right\|_{h} \\
& +\left|b_{\text {lift }}\left(\mathbf{u}-\mathbf{u}_{h}, \xi_{\psi}\right)\right|+R_{1}+R_{2}+R_{3},
\end{aligned}
$$


where we isolated the term $b_{\text {lift }}\left(\mathbf{u}-\mathbf{u}_{h}, \xi_{\psi}\right)$ that needs to be treated separately. By the Cauchy-Schwarz inequality we have

$$
\begin{aligned}
&\left|b_{\text {lift }}\left(\mathbf{u}-\mathbf{u}_{h}, \xi_{\psi}\right)\right|^{2} \\
&=\left|k^{2} \int_{\Omega} \xi_{\psi} \nabla_{h} \cdot\left(\mathbf{u}-\mathbf{u}_{h}\right) d \mathbf{x}\right|^{2}+\left|k^{2} \int_{\Omega} \mathcal{M}\left(\llbracket \mathbf{u}-\mathbf{u}_{h} \rrbracket_{N}\right) \xi_{\psi} d \mathbf{x}\right|^{2} \\
& \leq C^{2}\left(k^{2} \beta \sum_{K \in \mathcal{T}_{h}} h_{K}^{2}\left\|\nabla_{h} \cdot\left(\mathbf{u}-\mathbf{u}_{h}\right)\right\|_{0, K}^{2}+k^{2} \beta\left\|\mathcal{M}\left(\mathrm{h} \llbracket \mathbf{u}-\mathbf{u}_{h} \rrbracket_{N}\right)\right\|_{0, \Omega}^{2}\right) \\
& \cdot\left(k^{2} \beta^{-1} \sum_{K \in \mathcal{T}_{h}} h_{K}^{-2}\left\|\xi_{\psi}\right\|_{0, K}^{2}\right),
\end{aligned}
$$

with $C=C(\kappa)$. Now, using the approximation property of Lemma 22 of the Clément operator, the third estimate in Proposition 12, the second estimate in (20) and the definition of $\left\|\left(\mathbf{u}-\mathbf{u}_{h}\right)\right\|_{h}$, we get

$$
\begin{aligned}
& \left|b_{\text {lift }}\left(\mathbf{u}-\mathbf{u}_{h}, \xi_{\psi}\right)\right|^{2} \\
& \leq C^{2} k^{2} \beta^{-1}\left(k^{2} \beta \sum_{K \in \mathcal{T}_{h}} h_{K}^{2}\left\|\nabla_{h} \cdot\left(\mathbf{u}-\mathbf{u}_{h}\right)\right\|_{0, K}^{2}+k^{2} \beta\left\|\mathrm{h}^{\frac{1}{2}} \llbracket \mathbf{u}-\mathbf{u}_{h} \rrbracket_{N}\right\|_{0, \mathcal{E}_{\mathcal{I}}}^{2}\right)\|\psi\|_{1, \Omega}^{2} \\
& \leq C^{2} k^{2} \beta^{-1}\left\|\left(\mathbf{u}-\mathbf{u}_{h}, 0\right)\right\|_{h}^{2}\left\|\mathbf{u}-\mathbf{u}_{h}\right\|_{0, \Omega}^{2},
\end{aligned}
$$

where $C=C\left(\kappa, \ell, C_{\text {ell }}\right)$. Inserting this, (22) and the estimates for the residuals obtained in Step 2 in (23) completes the proof.

The proof of Theorem 7 follows now from Corollary 25 and Proposition 26.

\section{Conclusions}

In this paper, we have carried out an error analysis for stabilized interior penalty discontinuous Galerkin methods for the discretization of the indefinite time-harmonic Maxwell equations. We have derived error estimates that are optimal, provided that the stabilization parameters are large enough and the meshsize is small enough. A numerical study of the proposed methods is the subject of ongoing work.

\section{Acknowledgment and disclaimer}

The effort of Peter Monk was sponsored by the Air Force Office of Scientific Research, Air Force Materials Command, USAF, under grant number F49620-96-10039. The US Government is authorized to reproduce and distribute reprints for governmental purposes notwithstanding any copyright notation thereon. The views 
and conclusions contained herein are those of the authors and should not be interpreted as necessarily representing the official policies or endorsements, either expressed or implied, of the Air Force Office of Scientific Research or the US Government.

\section{References}

[1] F. Ihlenburg, I. Babuška, Finite element solution of the Helmholtz equation with high wavenumber, Part I: The h-version of the FEM, Comput. Math. Appl. 30 (1995) 9-37.

[2] F. Ihlenburg, I. Babuška, Finite element solution of the Helmholtz equation with high wave number, Part II: The h-p version of the FEM, SIAM J. Numer. Anal. 34 (1997) $315-358$.

[3] M. Costabel, M. Dauge, Singularities of electromagnetic fields in polyhedral domains, Arch. Ration. Mech. Anal. 151 (2000) 221-276.

[4] M. Dauge, M. Costabel, D. Martin, Numerical investigation of a boundary penalization method for Maxwell equations, Preprint (1999).

[5] M. Dauge, M. Costabel, D. Martin, Weighted regularization of Maxwell equations in polyhedral domains, Preprint (2001).

[6] J. Nédélec, Mixed finite elements in $\mathbb{R}^{3}$, Numer. Math. 35 (1980) 315-341.

[7] J. Nédélec, A new family of mixed finite elements in $\mathbb{R}^{3}$, Numer. Math. 50 (1986) 57-81.

[8] J.-M. Jin, The finite element method in electromagnetics, Wiley, 1993.

[9] R. Silvester, R. Ferrari, Finite element methods for electrical engineers, 3rd Edition, Cambridge University Press, 1996.

[10] P. Monk, A finite element method for approximating the time-harmonic Maxwell equations, Numer. Math. 63 (1992) 243-261.

[11] L. Demkowicz, P. Monk, Discrete compactness and the approximation of Maxwell's equations in $\mathbb{R}^{3}$, Math. Comp. 70 (2001) 507-523.

[12] D. Boffi, Fortin operator and discrete compactness for edge elements, Numer. Math. 87 (2000) 229-246.

[13] A. Bossavit, Mixed finite elements and the complex of Whitney forms, in: J. Whiteman (Ed.), The Mathematics of Finite Elements and Applications VI, Academic Press, 1988, pp. 137-144.

[14] R. Hiptmair, Canonical construction of finite elements, Math. Comp. 68 (1999) 13251346.

[15] R. Hiptmair, Discrete Hodge-operators: An algebraic perspective, Journal of Electromagnetic Waves and Applications 15 (2001) 343-344. 
[16] L. Vardapetyan, L. Demkowicz, $h p$-adaptive finite elements in electromagnetics, Comput. Methods Appl. Mech. Engrg. 169 (1999) 331-344.

[17] J. Douglas, Jr., T. Dupont, Interior penalty procedures for elliptic and parabolic Galerkin methods, Vol. 58 of Lecture Notes in Physics, Springer-Verlag, Berlin, 1976.

[18] G. Baker, Finite element methods for elliptic equations using nonconforming elements, Math. Comp. 31 (1977) 45-59.

[19] M. Wheeler, An elliptic collocation-finite element method with interior penalties, SIAM J. Numer. Anal. 15 (1978) 152-161.

[20] D. Arnold, An interior penalty finite element method with discontinuous elements, SIAM J. Numer. Anal. 19 (1982) 742-760.

[21] P. Houston, C. Schwab, E. Süli, Discontinuous $h p$ finite element methods for advection-diffusion problems, Tech. Rep. NA 00-15, Oxford University Computing Laboratory (2000).

[22] B. Rivière, M. F. Wheeler, V. Girault, A priori error estimates for finite element methods based on discontinuous approximation spaces for elliptic problems, SIAM J. Numer. Anal., accepted for publication.

[23] B. Rivière, M. Wheeler, V. Girault, Part I: Improved energy estimates for interior penalty, constrained and discontinuous Galerkin methods for elliptic problems, Computational Geosciences 3 (4) (1999) 337-360.

[24] B. Cockburn, C.-W. Shu, The local discontinuous Galerkin method for time-dependent convection-diffusion systems, SIAM J. Numer. Anal. 35 (1998) 2440-2463.

[25] P. Castillo, B. Cockburn, I. Perugia, D. Schötzau, An a priori error analysis of the local discontinuous Galerkin method for elliptic problems, SIAM J. Numer. Anal. 38 (2000) 1676-1706.

[26] C. Baumann, J. Oden, A discontinuous $h p$-finite element method for convectiondiffusion problems, Comput. Methods Appl. Mech. Engrg. 175 (1999) 311-341.

[27] J. Oden, I. Babuška, C. Baumann, A discontinuous $h p$ finite element method for diffusion problems, J. Comput. Phys. 146 (1998) 491-519.

[28] D. Arnold, F. Brezzi, B. Cockburn, L. Marini, Unified analysis of discontinuous Galerkin methods for elliptic problems, Tech. Rep. 1747, IMA, University of Minnesota (2001).

[29] S. Prudhomme, F. Pascal, J. Oden, A. Romkes, Review of a priori error estimation for discontinuous Galerkin methods, Tech. Rep. 2000-27, TICAM, University of Texas at Austin (2000).

[30] I. Perugia, D. Schötzau, The $h p$-local discontinuous Galerkin method for lowfrequency time-harmonic Maxwell's equations, Tech. Rep. 1774, IMA, University of Minnesota, submitted (2001). 
[31] O. Cessenat, Application d'une nouvelle formulation variationnelle aux équations d'ondes harmoniques. Problèmes de Helmholtz 2D et de Maxwell 3D, Ph.D. thesis, Université Paris IX Dauphine (1996).

[32] F. Ben Belgacem, A. Buffa, Y. Maday, The mortar method for the Maxwell's equations in 3D, C. R. Acad. Sci. Paris, Série I 329 (1999) 903-908.

[33] A. Buffa, Y. Maday, F. Rapetti, A sliding mesh-mortar method for a two dimensional eddy currents model of electric engines, Math. Models Appl. Sci. 35 (2001) 191-228.

[34] C. Wolfe, U. Navsariwala, S. Gedney, A parallel finite-element tearing and interconnecting algorithm for solution of the vector wave equation with PML absorbing medium, IEEE Transactions on Antennas and Propagation 48 (2000) 278284.

[35] Z. Chen, Q. Du, J. Zou, Finite element methods with matching and nonmatching meshes for Maxwell equations with discontinuous coefficients, SIAM J. Numer. Anal. 37 (2000) 1542-1570.

[36] S. Caorsi, P. Fernandes, M. Raffetto, On the convergence of Galerkin finite element approximations of electromagnetic eigenproblems, SIAM J. Numer. Anal. 38 (2000) 580-607.

[37] C. Amrouche, C. Bernardi, M. Dauge, V. Girault, Vector potentials in threedimensional non-smooth domains, Math. Models Appl. Sci. 21 (1998) 823-864.

[38] P. Ciarlet, The Finite Element Method for Elliptic Problems, North-Holland, Amsterdam, 1978.

[39] J. L. Lions, E. Magenes, Problèmes aux Limites Non-Homogènes et Applications, Dunod, Paris, 1968.

[40] B. Cockburn, G. Kanschat, D. Schötzau, C. Schwab, Local discontinuous Galerkin methods for the Stokes system, Tech. Rep. 214, Minnesota Supercomputing Institute, University of Minnesota, submitted (2000).

[41] P. Castillo, Performance of discontinuous Galerkin methods for elliptic partial differential equations, Tech. Rep. 1764, IMA, University of Minnesota (2001).

[42] A. Schatz, An observation concerning Ritz-Galerkin methods with indefinite bilinear forms, Math. Comp. 28 (74) 959-962.

[43] L. Franca, T. Hughes, R. Stenberg, Stabilized finite element methods, in: M. Gunzburger, R. Nicolaides (Eds.), Incompressible Computational Fluid Dynamics: Trends and Advances, Cambridge University Press, 1993, pp. 87-107.

[44] V. Girault, P. Raviart, Finite Element Approximations of the Navier-Stokes Equations, Springer Verlag, New York, 1986.

[45] A. Alonso, A. Valli, An optimal domain decomposition preconditioner for lowfrequency time-harmonic Maxwell equations, Math. Comp. 68 (1999) 607-631. 